NISTIR 6939

\title{
MatML Version 3.0 Schema
}

E.F. Begley

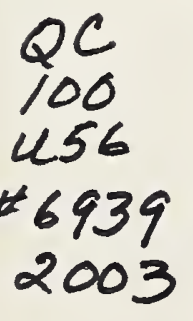




\title{
MatML Version 3.0 Schema
}

\author{
E.F. Begley \\ Building Environment Division \\ Building and Fire Research Laboratory
}

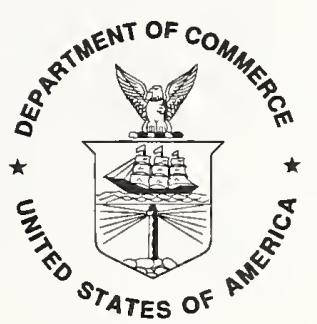

U.S. DEPARTMENT OF COMMERCE Donald L. Evans, Secretary TECHNOLOGY ADMINISTRATION

Phillip J. Bond, Under Secretary of Commerce for Technology NATIONAL INSTITUTE OF STANDARDS AND TECHNOLOGY Arden L. Bement, Jr., Director 



\section{Introduction}

\section{What is MatML?}

On 01 October 1999, the National Institute of Standards and Technology (NIST) initiated the development of MatML, a markup language for the management and exchange of materials property data. At NIST, the need for such a markup language became evident in May 1997 during the development of the Ceramics WebBook ${ }^{1}$, which includes databases of evaluated data for advanced ceramics, a guide to materials data centers and sources, and a collection of materials-related tools and resources. It was during the May 1999 ASTM-NIST Workshop on Materials Data in the Internet Age, however, when it became clear there were many independent realizations that a materials data markup language was needed. A few months later, NIST addressed this need by launching the MatML development project. The first order of business was the assembly of an international working group composed of materials data experts. The MatML Working Group's central activities were to determine the scope of MatML's application and to formulate its vocabulary and syntax. There was an especially strong emphasis during the working group discussions that: MatML ought to be flexible in the scope of its application, i.e., that it could be used to markup a wide variety of materials data from a broad range of sources; MatML's vocabulary should be materials-oriented and easy to learn; and MatML's syntax must be extensible to accommodate both alternative and unanticipated forms of materials data and their representation.

Perhaps the best way to describe MatML is by example and in contrast to HTML. Materials property data distributed on the World Wide Web in documents using hypertext markup language (HTML) present two problems for computer applications intending to use the data: interpretation and interoperability.

Figure 1 contains an HTML code fragment for

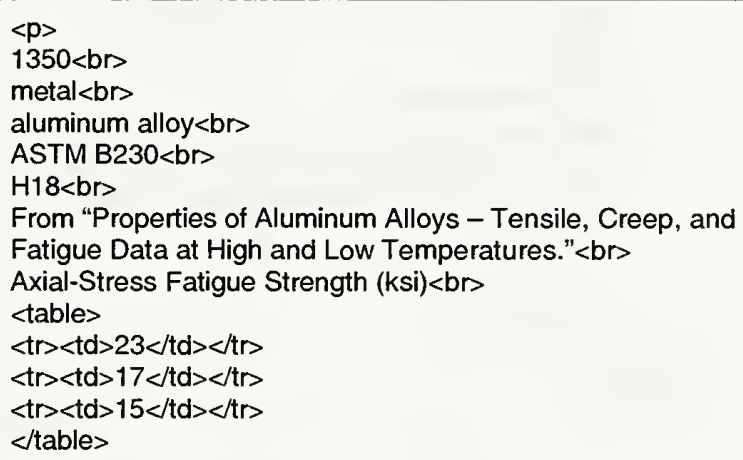

Fig. 1: Aluminum alloy data in HTML

aluminum alloy data. Since the data are not self-describing, one would need a complete data dictionary and glossary of terms as well as exact detailed knowledge of the document structure in order to write a computer application that uses these data without human intervention. In Figure 1, for example, it would be necessary to distinguish the descriptors of the material (1350, metal, aluminum alloy, ASTM B230, H18) from the property data (table of axial-stress fatigue strengths in ksi units). Assuming a consistent structure within and across documents from the data source, it would then be possible to parse and interpret those data programmatically for subsequent use in another computer application. In absence of tightly and consistently structured documents, a data dictionary, and a glossary of terms, however, interpretation arises as a key stumbling block for automated use of the materials property data contained in HTML documents.

If interpretation of data were not an issue, the computer application developed to parse and use the data would likely encounter serious difficulties were it to try using data from other structurally dissimilar sources. Interoperability, then, would be a limiting condition for the use of the computer application across structurally dissimilar data sources even if data dictionaries and glossaries of terms were available.

MatML addresses the problems of interpretation and interoperability for materials property data exchanged via the World Wide Web. The example given in Figure 1 might be coded as in Figure 2 and would allow an application to interpret and use materials data regardless of their sources.

\footnotetext{
${ }^{1}$ See: http://www.ceramics.nist.gov/webbook/webbook.htm
} 
HTML tags specify only how the data are to be formatted for display and convey no description of the data themselves. While this aspect of HTML has enabled the publication of billions of web documents, it,

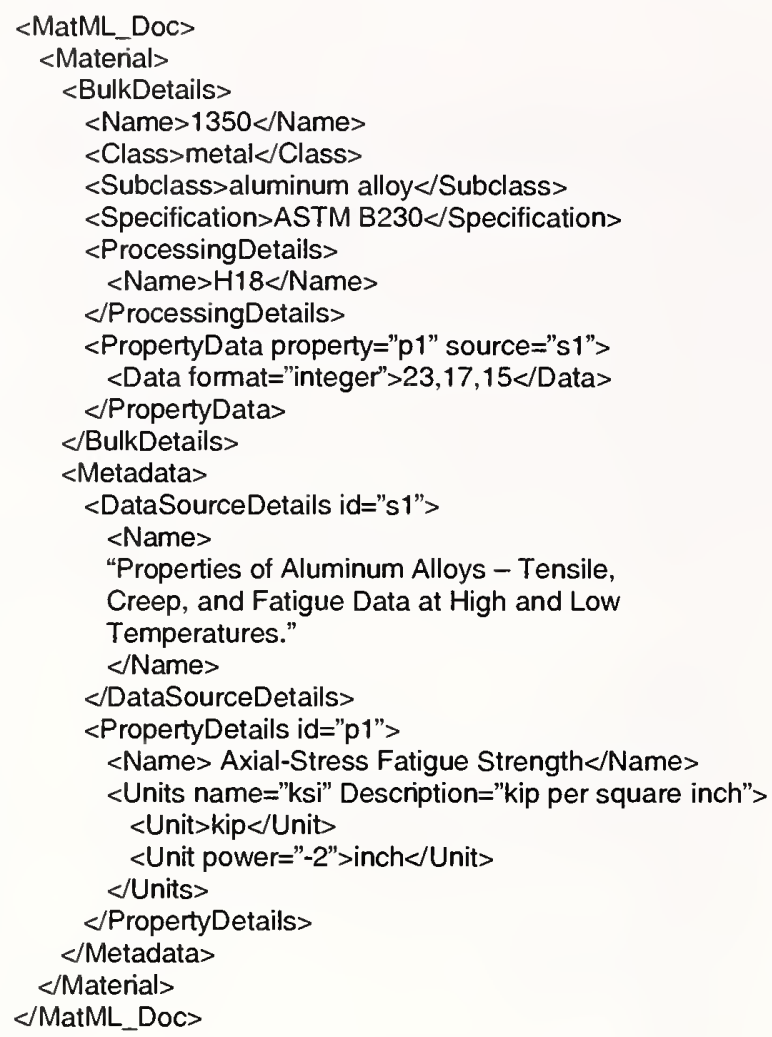

Fig. 2: Aluminum alloy data in MatML nonetheless, represents a serious drawback to those who wish to automate the processing of the data contained in those documents. To address this problem, the Extensible Markup Language ${ }^{2}$ (XML) was developed and released by the World Wide Web Consortium ${ }^{3}$ (W3C), an international group of approximately 500 member organizations dedicated to developing common protocols that promote the evolution of the Web and ensure its interoperability.

XML enables communities, such as materials scientists and engineers in the case of MatML, to define their own domain-specific tags and document structure, i.e., they can create their own languages for data management and exchange that, in turn, permit efficient parsing and interpretation of those data via software.

The descriptive nature of the MatML tags, such as $<$ Name $>,<$ Class $>$, and $<$ Subclass $>$ is plainly evident, permitting the language to be far more intelligible than non-descriptive fixed tagsets such as HTML. At the same time, MatML defines a coherent and consistent document structure for its tags, which ensures that any programming language can be used to parse and process the data in whatever manner required. The formal specification for MatML 3.0 Schema, which covers syntax and semantics, is provided in Appendix A.

\section{MatML Development History}

The most important attribute of the MatML development effort was the inclusion of a broad range of input from the materials community. These individuals and groups represented private industry, government laboratories, universities, standards organizations, and professional societies, and their efforts were coordinated by NIST.

The initial strategy for MatML's development included the establishment of a working group (Table 1) that delineated the scope and specifications for the new language from which MatML was developed. On 28 April 2000, an initial working draft of the language, Annotated MatML DTD Version 1.0 (MatML 1.0), was released for comment. In response to working group feedback, a second version of MatML, Annotated MatML DTD Version 2.0 (MatML 2.0), was released on 29 March 2001. A few months later, on 26-27

\footnotetext{
${ }^{2}$ See: http://www.w3.org/XML

${ }^{3}$ See: http://www.w3.org/Consortium/
} 


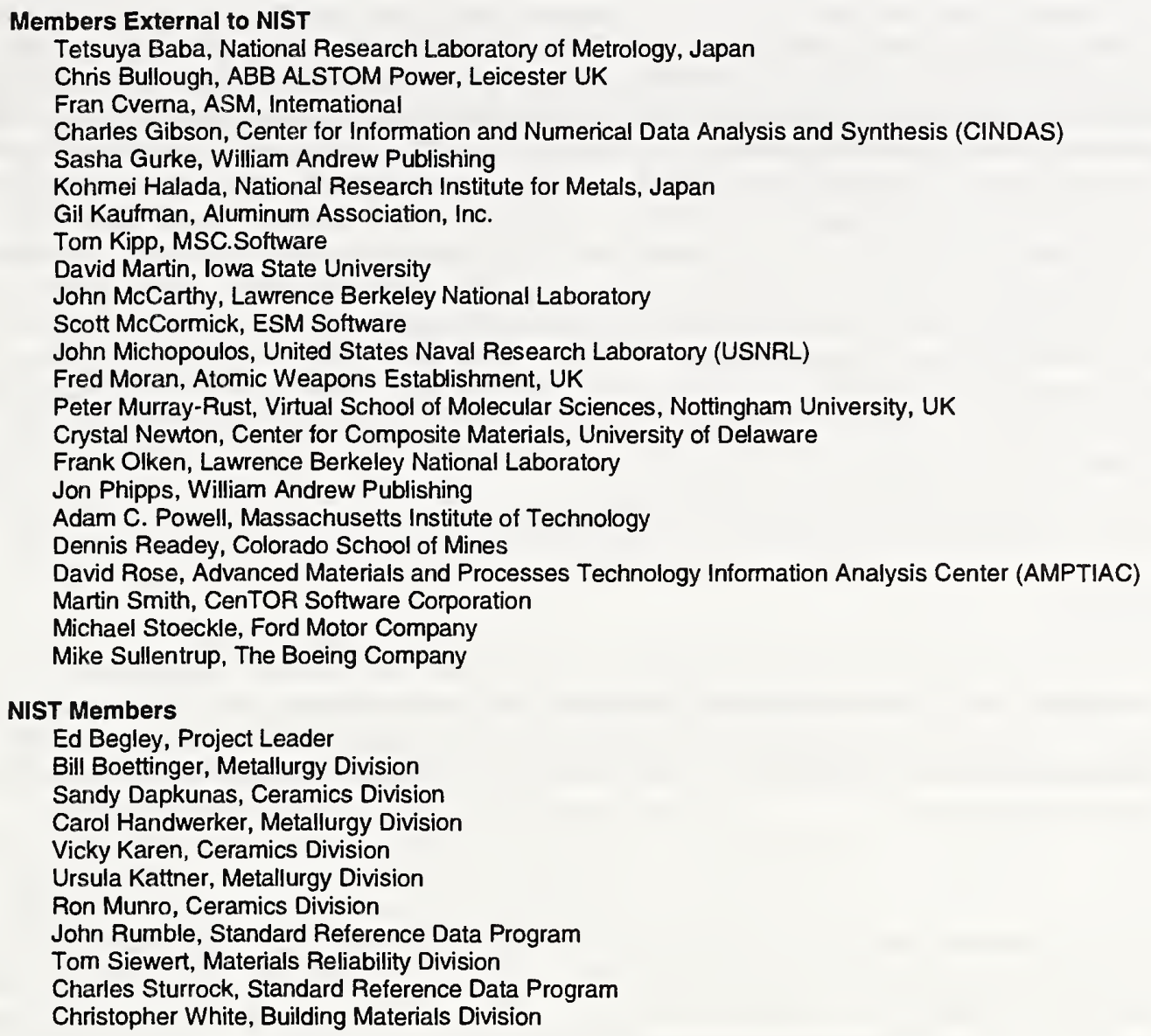

Table 1: MatML Working Group (1999-2001)

June 2001, the Workshop on the Technical and Strategic Future of MatML ${ }^{4}$ was hosted by NIST during which open discussions were held to tabulated specific issues that required further technical refinement of the language. MatML 3.0 Schema (MatML 3.0) is an enhancement of MatML 2.0 that addresses the technical issues identified during the workshop.

\section{Approach}

The specific revisions to MatML 2.0 resulting in MatML 3.0 include: porting the language specification from XML DTD to XML Schema; eliminating redundant entry of specific types of information; overhauling the Units content model; providing a mechanism for encoding measurement uncertainty; revising the content model for Terms; modifying several element content models to improve support for encoding data for composites; and addressing a reported document validation difficulty.

\section{Schema}

From the outset of the MatML project, the working group suggested that the specification for the language, ultimately, ought to be prepared using the XML Schema Definition Language (XML Schema) rather than

\footnotetext{
${ }^{4}$ Sturrock, C.P., Begley, E.F., and Kaufman, J.G., "NISTIR 6785 MatML - Materials Markup Language Workshop Report," National Institute of Standards and Technology, Gaithersburg, MD, August 2001.

${ }^{5}$ See: http//www.w3.org/2001/XMLSchema
} 
XML Document Type Definition (DTD). The reasons for using XML Schema include obviating notable shortcomings of DTD's and, in anticipation of MatML's acceptance testing phase, providing an improved language for software development. XML Schema, for example, provides robust support for data typing, including user-defined data types, whereas DTD's treat all data content essentially as text. Support for data types makes it easier to define permissible data values and other restrictions, to validate data, and to convert data between different data types. All of these advantages are, among other applications, essential for working with data from databases. XML Schemas, furthermore, are written in XML whereas DTD's are not. This is especially significant since it means that: XML editors and parsers can be used to edit and parse Schema files; Schemas can be manipulated using the XML Document Object Model $^{6}$ (DOM) (The DOM views the document as a collection of objects that can be manipulated, i.e., items can be added, deleted, and modified programmatically.); and Schemas can be transformed using XML stylesheet language transformations ${ }^{7}$ (XSLT) (XSLT is used to transform and format documents for display.) Since the MatML development effort was initiated before the W3C had formally approved XML Schema as a recommendation, it was decided, for the short term, to define MatML 1.0 and MatML 2.0 using DTD's. On 02 May 2001, the W3C approved XML Schema 1.0. In response, MatML has been rewritten and the new version conforms to the XML Schema Definition Language.

\section{Redundancy}

Sample instance documents encoded using MatML 2.0 illustrated that descriptions of properties, measurement techniques, data sources, and parameters could be repeated many times throughout a document. This issue has been addressed by the introduction of the Metadata element, which, in addition to the previously mentioned descriptions, includes another new element, SpecimenDetails, for encoding descriptions of specimens.

\section{Units}

MatML 2.0 represented units as a simple string and permitted embedded HTML tags. MatML 3.0 uses the Units content model developed by Dr. Edward J. Shaya of NASA Goddard Space Flight Center for the eXtensible Data Format (XDF) language version $0.17^{8}$. This improved representation of units will permit software developers to implement units conversion applications.

\section{Uncertainty}

Previous versions of MatML did not provide for measurement uncertainty. MatML 3.0 includes a new element, Uncertainty, for encoding this information and was constructed based upon the recommendations of Dr. Ron Munro of NIST's Ceramics Division (MSEL) and Dr. Raghu Kacker of NIST's Mathematical and Computational Sciences Division in the Information Technology Laboratory.

\section{Glossary}

The Terms element in MatML 2.0 has been replaced by the Glossary element in MatML 3.0, which uses a simplified content model for encoding terms instead of the Virtual HyperGlossary ${ }^{(\mathrm{TM})} 9$ markup language.

\section{Composites}

MatML 3.0 includes the revisions proposed by the MatML Composites Working Group. These changes include recursion of the ComponentDetails element as well as new attributes on the Material element (id, layers, local_frame_of_reference) and the ComponentDetails element (id).

\footnotetext{
${ }^{6}$ See: http://www.w3.org/DOM/

${ }^{7}$ See: http://www.w3.org/TR/xslt

${ }^{8}$ See: http://xml.gsfc.nasa.gov/DTD/XDF_017.dtd

${ }^{9}$ See: http://www.vhg.org.uk/home/index_frame.html
} 
Nesting

The MatML ASM-MSC Data Exchange Focus Group reported difficulties validating MatML 2.0 instance documents where multiple element tuples ("tuple" is a data object containing two or more data elements) were encoded within a single element (Compound, PropertyDetails, etc.). Multiple element tuples have been eliminated in MatML 3.0 and have been replaced by nested content models.

\section{Results}

Appendix A contains the formal specification for MatML Version 3.0 Schema, including detailed documentation of each element's content model. The following discussion presents a high-level overview of the language specification.

MatML_Doc

The root element for a MatML document is named MatML_Doc. The MatML_Doc element contains one or more Material elements, each of which describes a material and its properties.

\section{Material}

The information contained by the Material element is compartmentalized into five major elements:

1. BulkDetails element contains a description of the bulk material;

2. ComponentDetails element contains a description of each component of the bulk material (useful for complex materials systems such as composites or welds);

3. Metadata element contains descriptions of data found in the document;

4. Graphs element encodes two dimensional graphics;

5. Glossary element contains definitions of terms found in the document.

\section{BulkDetails}

The BulkDetails element is composed of the following elements, which are fully documented in Appendix A:

- Name contains the bulk material's name;

- Class contains the bulk material's class;

- Subclass contains the bulk material's subclass;

- Specification contains the bulk material's specification;

- Source contains the source of the bulk material;

- Form contains the form of the bulk material;

- ProcessingDetails contains a description of a step in the processing history of the bulk material;

- Geometry contains a description of the geometry of the bulk material;

- Characterization contains the characterization of the bulk material including the chemical formula, chemical composition, phase composition, and dimensional details;

- PropertyData contains data for a property of the bulk material;

- Notes contains any additional information concerning the bulk material.

\section{ComponentDetails}

The ComponentDetails element is composed of the following elements, which are fully documented in Appendix A:

- Name contains the component's name;

- Class contains the component's class;

- Subclass contains the component's subclass;

- Specification contains the component's specification;

- Source contains the source of the component;

- Form contains the form of the component; 
- ProcessingDetails contains a description of a step in the processing history of the component;

- Geometry contains a description of the geometry of the component;

- Characterization contains the characterization of the component, including the chemical formula, chemical composition, phase composition, and dimensional details;

- PropertyData contains data for a property of the component;

- AssociationDetails contains a description of the relationship of the component to another component;

- Notes contains any additional information concerning the component;

- ComponentDetails contains a description of a component within the component.

\section{Metadata}

The Metadata element contains descriptions of the data sources, properties, measurement techniques, specimens, and parameters encoded within the MatML document. The Metadata element is composed of the following elements, which are fully documented in Appendix A:

- DataSourceDetails contains a description of a data source;

- PropertyDetails contains a description of a property;

- MeasurementTechniqueDetails contains a description of a measurement technique;

- SpecimenDetails contains a description of a specimen;

- ParameterDetails contains a description of a parameter.

\section{Graphs}

The Graphs element uses the W3C's Scalable Vector Graphics ${ }^{10}$ markup language (SVG) for describing two dimensional graphics and allows for three types of graphical objects: vector graphics shapes, images, and text.

\section{Glossary}

The Glossary element contains terms, each of which is encoded using the Term element. The Term element is composed of the following elements, which are fully described in Appendix A:

- Name contains the term's name;

- Definition contains the term's definition;

- Abbreviation contains the term's abbreviation(s);

- Synonym contains the term's synonym(s);

- Notes contains any additional information pertinent to the term.

\section{Discussion}

With the publication of MatML 3.0, it is now possible to address other main findings of the 2001 MatML workshop. These include:

- A good set of illustrations demonstrating the power and flexibility of MatML should be established in order to advance the widespread adoption of MatML (Appendix B);

- MatML must be tested thoroughly, involving individuals at various stages in the material development/application cycle ranging from materials specialists who select materials to designers responsible for addressing load-carrying capacity and failure limits. In addition, the needs of materials scientists exploring the fundamental structure and properties of materials, and of journal publishers disseminating materials information, must also be addressed;

${ }^{10}$ See: http://www.w3.org/TR/SVG/ 
- At the appropriate time MatML should be recognized officially and formally via registry with XML repositories such as xml.org and/or by standards organizations such as the Object Management Group, ASTM, or ANSI;

- The overall effort should be linked with key industries, that might, via some of their applications, be in position to provide good tests for MatML and, assuming success, encourage or perhaps even require users of their information systems to use MatML as the data exchange medium.

J.G. Kaufman, Chairman, The Aluminum Association, Inc. (retired Vice President) Ed Begley, Lead Technical Expert, NIST

Fran Cvema, ASM Intemational

Doug Fleming, Automation Creations, Inc.

Chris Grethlein, AMPTIAC

Scott McCormick, ESM Software

Debbie Mies, MSC Software

Michael Mitchell, NASA Marshall Space Flight Center

Chris Nunez, CenTOR Software Corporation

Kent A. Reéd, BFRL, NIST

James D. Rossi, Westmoreland Testing \& Research, Inc.

Craig Seymour, Granta Design Limited, UK

Charles Sturrock, MSEL, NIST

Mike Sullentrup, The Boeing Company

Table 2: MatML Steering Committee (2002)

To address some of the key strategic needs among these findings, the MatML

Steering Committee (Table

2) was formed during the autumn of 2001. The principal activities of the committee have been promotion of MatML including information dissemination, coordination of acceptance testing activities, and exploration of opportunities for MatML's long-term stewardship.

The MatML Steering Committee represents the interests of a much larger community of materials and computing professionals interested in MatML and its objective of automating the exchange of materials property data over the Web.

\section{Conclusion}

At present, the materials data "marketplace" can be very chaotic and difficult for both providers and consumers. Until the arrival of MatML, there was no common exchange format but instead hundreds of proprietary formats that resulted in wasteful duplication of effort and poor scale-up. Also plaguing the materials data community is the absence of software interoperability, whereby one computer program, such as a finite element code, can automatically input material properties from another computer program or

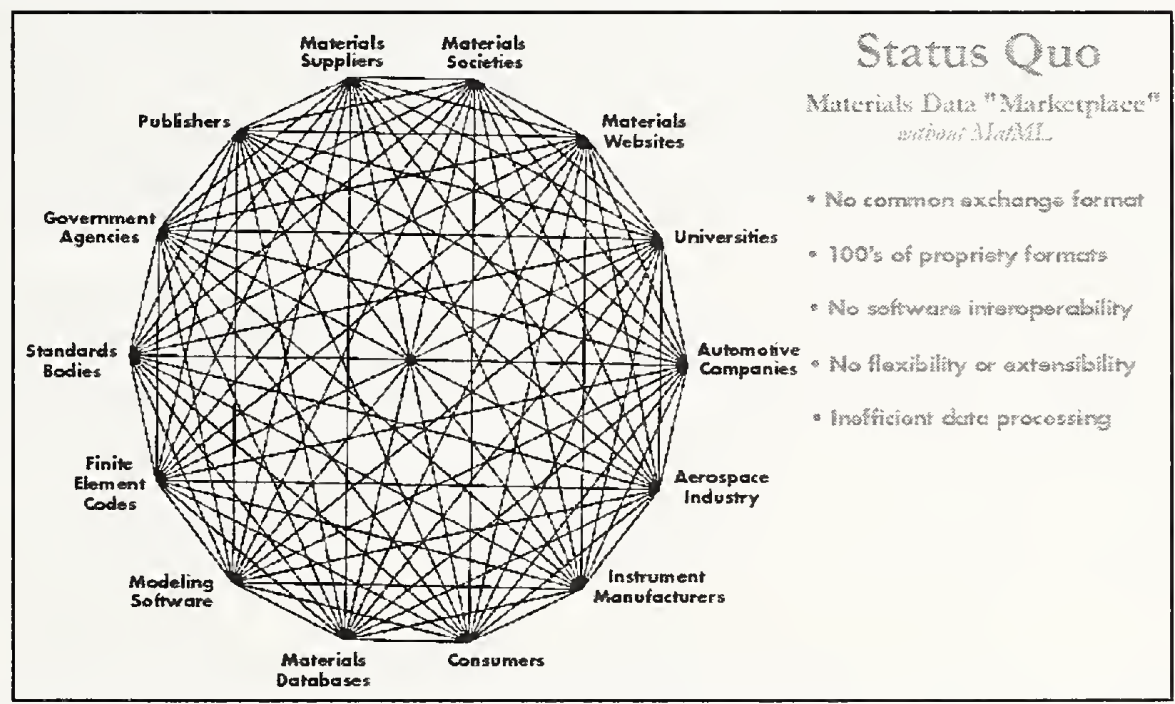
database without the necessity of human intervention. The present situation overall yields very inefficient data processing. 


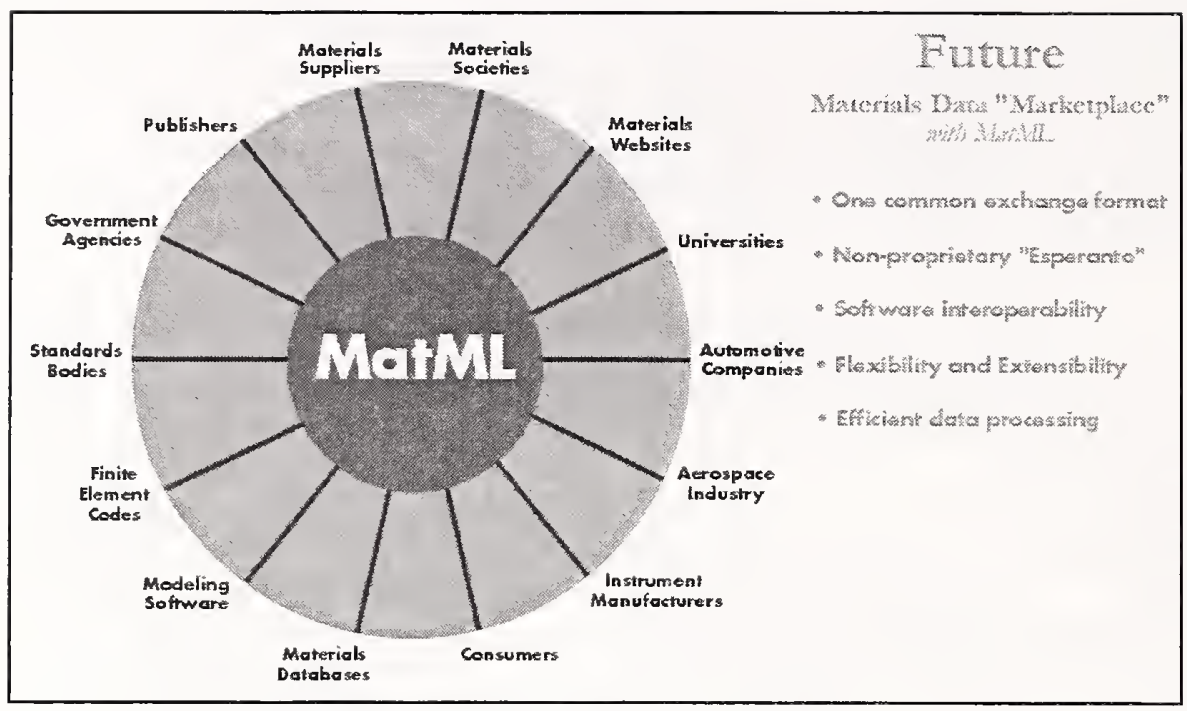

MatML will serve the materials data marketplace as a common, publicdomain materials data exchange format - a non-proprietary and generic language for materials property data. With considerable built-in flexibility and extensibility as attributes of the language, MatML will provide for direct program-to-program interoperability,

efficient data processing, and rapid, reliable, and useful response to searches for materials data over the Web. Its widespread adoption will provide for powerful and malleable connection of the various materials data sources found on the Web today, as well as those to be added to the Web tomorrow. 
APPENDIX A: MatMLVersion 3.0 Schema

\section{$<$ ? $x \mathrm{ml}$ version $=" 1.0$ " encoding $=" U T F-8$ "? $>$}

$<!-$

Tive

MatML Version 3.0 Schema

Author

This document was prepared by E.F. Begley (begley@ nist.gov) on behalf of the Math Working Group

(1999-2001) and the MatML Steering Commitee (2002).

\section{Copyrights}

This work is an official contribution of the National Institute of Standards and Technology (NIST) and is not subject to copyright in the United States.

"METADATA" is a trademark of the Metadata Company. Any use of the term "metadata" in this document is in a descriptive sense, meaning "data about data. "Mathl is not in any way atêliated with the Metadata Company.

\section{Acknowledgements}

The MatML development eftor has provided deepiy satisting personal and professional challenges for the author. He gratefully acknowledges the contributions of his collaborators.

Funding

This project was funded [in part] by NIST's Systems Integration for Manufacturing Applications

(SIMA) Program. SIMA supports NIST projects applying information technologies and

standards-based approaches to manufacturing software integration problems.

Project Support

Standard Reference Data Program (SRD), NIST

Materials Science and Engineering Laboratory (MSEL), NIST

Bulling and Fire Research Laboratoy (BFRL), NIST

Technical Collaboration

The MatML Working Group (1999-2001)

Members External to NIST

Tetsuya Baba, National Research Laboratory of Metrology, Japan

Chris Bullough, ABB ALSTOM Power, Leicester UK

Fran Cverna, ASM, Intemational

Charles Gibson, Center for Information and Numerical Data Analysis and Synthesis (CINDAS)

Sasha Gurke, Willam Andrew Publishing

Kohmei Halada, National Research Institute for Metals, Japan

Gil Kauman, Auminum Association. Inc.

Tom Kipp, MSC.Sofware

David Martin, lowa State Universily

John McCarthy, Lawrence Bekeley National Laboratory

Scot McCormick, ESM Sotware

John Michopoulos, United States Naval Research Laboraton (USNRL)

Fred Moran. Atomic Weapons Establishment, UK

Peter Murray-Rust. Vitual School of Molecular Sciences, Nottingham University, UK

Crystal Newton, Center for Composite Materials, University of Delaware

Frank Oken, Lawrence Berkeley National Laboratony

Jon Phipps, Willam Andrew Publishing

Adam C. Powell, Massachusetts Institute of Technology

Dennis Readey. Colorado School of Mines

David Rose, Advanced Materials and Processes Technology Information Analysis Center (AMPTIAC)

Martin Smith, CenTOR Soltware Corporation

Michael Stoeckle, Ford Motor Company

Mike Sullentrup, The Boeing Company

NIST Members

Bill Boettinger, Metallurgy Division, MSE

Sandy Dapkunas, Ceramics Division, MSEL

Carol Handwerker, Metalurgy Division, MSEL 
Vicky Karen, Ceramics Division, MSEL

Ursula Kattner, Metallurgy Division, MSEL

Ron Munro, Ceramics Division, MSEL

John Rumble, SRD, NIST

Tom Siewert, Materials Reliability Division, MSEL

Charles Sturrock, SRD, NIST

Christopher White, Building Materials Division, BFRL

The MatML Steering Committee (2002)

Gil Kauman, Committee Chair, Aluminum Assoication, Inc.

Fran Cverna, ASM Internationa!

Doug Fleming, Automation Creations, Inc.

Chris Grethlein, AMPTIAC

Scott McCormick. ESM Software

Debbie Mies, MSC Software

Michael Mitchell, NASA Marshall Space Flight Center

Chris Nunez, CenTOR Software Coporation

Kent A. Reed, BFRL, NIST

James D. Rossi, Westmoreland Testing \& Research, Inc.

Craig Seymour, Granta Design Limited. UK

Chanles Sturrock. MSEL. NIST

Mike Sullentrup, The Boeing Company

The author especially thanks:

Jim Fowler, SIMA Program Manager (NIST), for funding and supporting the MatML effort:

Gil Kaufman, Vice President (retired), The Aluminum Assocation, Inc, for his leadership while chairing the MatML Steering Committee, for his insightful technical criticisms, for the pleasure of his collaboration, periodically interrupted, across three decades, and for his personal and professional guidance:

John Rumble, Measurement Services Division Chief (NIST), for his support, knowledge, insights, and generosity with his time;

Ron Munro, Physicist (MSEL), for the benefit of his wisdom and recommendations as well as his deep and broad knowledge of materials property data:

John Michopoulos, Senior Research Scientist (USNRL), for his support, enthusiasm, technica! insights and, particularly, for his early adoption of MatML for use via namespace in his

development of femML, the finite element modelng markup language (htp:/Www. istos. org/femML);

George Kelly, Bulding Environment Division Chief (BFRL) and Kent Reed, Computer Integrated Bulling Processes Group Leader (BFRL), for supporting the completion of this work.

Revisions

MatML Version 3.0 Schema (henceforth reterred to as MatML 3.0) is an enhancement of the previously released Annotated MatML. DTD Version 2.0 (henceforth referred to as MatML 2.0) and addresses the technical issues identified during the June 2001 NIST workshop entitled "The Technical and Strategic Future of MatML." In addition to these revisions, the author reorganized several content models and renamed some elements to improve the clarity of the language.

Schema

MatML 3.0 is expressed in XML Schema Definition Language and contorms to World Wide Web Consortium (W3C) hitp://Www. w3.org/2001/XMLSchema.

Redundancy

Sample instance documents encoded using MatML 2.0 illustrated that descriptions of properties, measurement techniques, data sources. and parameters could be repeated many times throughout a document. This issue has been addressed by the introduction of the Metadata element. which, in addition to the previously mentioned descriptions, includes another new element, SpecimenDetalls, sor encoding descriptions of specimens.

Units

MatML 2.0 represented units as a simple string and permitted embedded HTML tags. MatML 3.0 
uses the Units content model ceveloped by Dr. Edward J. Shaya of NASA Goddard Space Fight Center for the eXtensible Data Format (XDF) language version 0.17 found at

htip://xml.gsfc.nasa.gov/DTD/XDF_017.dtd.

Uncertainty

MatML 2.0 did not provide for measurement uncerainty. MatML 3.0 includes a new element,

Uncertainty, for encoding this information and was constructed based upon the recommendations

of Dr. Ron Munro of NIST's Ceramics Division (MSEL) and Dr. Raghu Kacker of NIST's

Mathematical and Computational Sciences Division in the Information Technology Laboraton.

Glossary

The Terms element in MatML 2.0 has been replaced by the Glossary element in Math 3.0, which

uses a simplified content model for encoding tems instead of the Virual HyperGlossary(TM)

markup language.

Composites

MathL 3.0 includes the revisions proposed by the MatML Composites Working Group. These

changes include recursion of the ComponentDetals element as well as new attributes on the

Material elemen (id, layers, local frame_ofreference) and the Componentbetails element (id).

Nesting

The MatML ASM-MSC Data Exchange Focus Group reported dinticulties validating MathL 2.0 instance

documents where multiple element tuples were encoded within a single element (Compound,

PropertyDetails, etc.). Multiple element tuples have been eliminated in MatuL 3.0 and have been

replaced by nested content models.

$\rightarrow$

<xsd:schema xmins:xsd="http://www.w3.org/2001/XMLSchema" elementFormDefault="qualified" attributeFormDefault="unqualified"> <xsd:element name="MatML Doc">

<xsd:annotation>

<xsd:documentation>

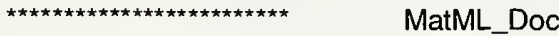

This element declares the content model for MatML_Doc, topmost in the hierarchy of elements that comprise a document marked up using MatML. Content models describe the relationships of the element and its child elements. MatML_Doc must contain one or more Material elements.

$</$ xsd:documentation $>$

$<$ xsd:annotation $>$

<xsd:complexType>

$<x$ sd:sequence $>$

<xsd:element ref="Material" maxOccurs="unbounded"/>

$<$ xsd:sequence $>$

$<$ xsd:complexType>

$<$ xsd:element>

<xsd:element name="Material">

$<$ <sd:annotation>

<xsd:documentation>

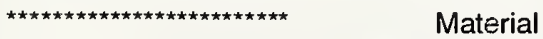

This element declares the content model for Material, which contains materials data. Material has three optional attributes.

The first attribute, id, may be used as an identification specifier for the material, which is especially useful for complex systems such as composite laminates.

The second attribute, layers, may be used to indicate the number of layers in complex systems such as composite laminates.

The third attribute, local_frame_of_reference, may be used as an identification specifier for the local material orientation relative to the 
global frame of reference, which is especially useful for complex systems such as anisotropic materials.

Material is composed of the following elements.

BulkDetails contains a description of the bulk material and must occur once and only once within the Material element. For additional information, see the documentation for the BulkDetails element.

ComponentDetails contains a description of a component within the bulk material and may occur zero or more times within the Material element. ComponentDetails may be used to describe complex materials systems such as welds (e.g. the base metal, the heat affected zone, and the weld metal) or composites (e.g. the whiskers, fibers, and matrix of a fiber-reinforced composite material). For additional information, see the documentation for the ComponentDetails element.

Metadata contains descriptions of the data sources, properties, measurement techniques, specimens, and parameters which are referenced when materials property data are encoded using the PropertyData element. Metadata may occur once or not at all within the Material element. For more information, see the documentation for the Metadata element.

Graphs contains descriptions of two dimensional graphics and may occur once or not at all within the Material element. For additional information, see the documentation for the Graphs element.

Glossary contains descriptions of the material and property terms used in the document and may occur once or not at all within the Material element. For additional information, see the documentation for the Glossary element.

$<$ xsd:documentation $>$

$</ x s d: a n n o t a t i o n>$

<xsd:complexType>

$<x s d$ :sequence $>$

$<x$ sd:element name="BulkDetails">

$<x$ d:annotation>

<xsd:documentation>

\section{BulkDetails}

This element declares the content model for BulkDetails, which contains a description of the bulk material and is composed of the following elements.

Name contains the material's name and has one optional attribute, authority, for identifying an authoritative source of material names. Name must occur once and only once within the BulkDetails element.

Class contains the material's class and may occur once or not at all within the BulkDetails element.

Subclass contains the material's subclass(es) and may occur zero or more times within the BulkDetails element.

Specification contains the material's specification(s) and has one optional attribute, authority, for identifying an authoritative source of material specifications. Specification may occur zero or more times within the BulkDetails element.

Source contains the name of the source of the material and may occur once or not at all within the BulkDetails element. 
Form contains the form of the material and may occur once or not at all within the BulkDetails element.

ProcessingDetails contains a description of a processing step for the material and may occur zero or more times within the BulkDetails element. For additional information, see the documentation for the ProcessingDetails element.

Geometry contains a description of the geometry of the material and may occur once or not at all within the BulkDetails element. For additional information, see the documentation for the Geometry element.

Characterization contains the characterization of the material, including the formula, chemical composition, phase composition, and dimensional details. Characterization may occur once or not at all within the BulkDetails element. For additional information, see the documentation for the Characterization element.

PropertyData contains the property data for the material and may occur zero or more times within the BulkDetails element. For additional information, see the documentation for the PropertyData element.

Notes contains any additional information concerning the bulk material and may occur once or not at all within the BulkDetails element.

$</$ xsd:documentation $>$

$<$ xsd:annotation $>$

<xsd:complexType>

<xsd:sequence>

$<x$ sd:element ref="Name"/>

<xsd:element ref="Class" minOccurs="0"/>

<xsd:element ref="Subclass" minOccurs="0" maxOccurs="unbounded"/>

$<x$ sd:element ref="Specification" minOccurs="0" maxOccurs="unbounded"/>

<xsd:element ref="Source" minOccurs="0"/>

$<x s d: e l e m e n t$ ref="Form" minOccurs $=" 0 " />$

<xsd:element ref="ProcessingDetails" minOccurs="0" maxOccurs="unbounded"/>

$<x s d: e l e m e n t$ ref="Geometry" minOccurs="0"/>

$<x s d$ :element ref="Characterization" minOccurs="0"/>

<xsd:element ref="PropertyData" minOccurs="0" maxOccurs="unbounded"/>

$<x s d: e l e m e n t$ ref $=$ "Notes" minOccurs="0"/>

$</ x$ sd:sequence $>$

$</$ xsd:complexType>

$<$ xsd:element

<xsd:element ref="ComponentDetails" minOccurs="0" maxOccurs="unbounded"/>

<xsd:element name="Metadata" minOccurs="0">

$<x s d$ :annotation>

<xsd:documentation>

\section{Metadata}

This element declares the content model for Metadata, which contains descriptions of data sources, properties, measurement techniques, specimens, and parameters, and is composed of the following elements

DataSourceDetails contains a description of a data source referenced using the PropertyData element and may occur zero or more times within the Metadata element. For additional information, see the documentation for the DataSourceDetails element.

PropertyDetails contains a description of a property for which materials data are encoded using the PropertyData element and may occur zero or more times within the Metadata element. For additional information, see the documentation for the PropertyDetails element. 
Measurement TechniqueDetails contains a description of a measurement technique referenced using the PropertyData element and may occur zero or more times within the Metadata element. For additional information, see the documentation for the MeasurementTechniqueDetails element.

SpecimenDetails contains a description of a specimen referenced using the PropertyData element and may occur zero or more times within the Metadata element. For additional information, see the documentation for the SpecimenDetails element.

ParameterDetails contains a description of a parameter referenced using the PropertyData element and may occur zero or more times within the Metadata element. For additional information, see the documentation for the ParameterDetails element.

$</$ xsd:documentation $>$

$</ x s d: a n n o t a t i o n>$

<xsd:complexType>

$<x s d$ :sequence $>$

<xsd:element name="DataSourceDetails" minOccurs="0" maxOccurs="unbounded" > <xsd:annotation>

$<x$ sd:documentation>

\section{DataSourceDetails}

This element declares the content model for DataSourceDetails, which contains a description of a data source referenced by the PropertyData element. DataSourceDetails has one required attribute, id, which may be arbitrarily assigned but must be unique among id attributes assigned elsewhere in a MatML document. DataSourceDetails also has one optional attribute, type, for specifying the type of the data source (examples include "unpublished report," "journal," "handbook," etc.) DataSourceDetails is composed of the following elements.

Name contains the name of the data source and has one optional attribute, authority, for identifying an authoritative source of data source names. Name must occur once and only once within the DataSourceDetails element.

Notes contains any additional information concerning the data source and may occur once or not at all within the DataSourceDetails element.

$</$ xsd:documentation $>$

$</ x s d: a n n o t a t i o n>$

<xsd:complexType>

$<x$ sd:sequence $>$

<xsd:element ref="Name"/>

$<x$ sd:element ref="Notes" minOccurs="0"/>

$<$ xsd:sequence $>$

$<x$ sd:attribute name="id" type="xsd:ID" use="required"/>

$<x$ sd:attribute name="type" type="xsd:string" use="optional"/>

$<$ xsd:complexType>

$</ x s d: e l e m e n t>$

<xsd:element name="PropertyDetails" minOccurs="0" maxOccurs="unbounded"> <xsd:annotation>

<xsd:documentation>

PropertyDetails

This element declares the content model for PropertyDetails, which contains a description of a property referenced by the PropertyData element. PropertyDetails has one required attribute, id, which may be arbitrarily assigned but must be unique among id attributes assigned elsewhere in a MatML document. PropertyDetails also has one optional attribute, type, for specifying the type of the property (examples include "thermal," "mechanical," "electrical," etc.) PropertyDetails is composed of 
the following elements.

Name contains the name of the property and has one optional attribute, authority, for identifying an authoritative source of property names.

Name must occur once and only once within the PropertyDetails

element.

Units and Unitless are mutually exclusive elements for describing the property's units. Units or Unitless must occur once and only once within the PropertyDetails element. For additional information, see the documentation for the Units and Unitless elements.

Notes contains any additional information concerning the property and may occur once or not at all within the PropertyDetails element.

$</ x s d:$ documentation $>$

$<$ xsd:annotation $>$

<xsd:complexType>

<xsd:sequence>

$<x$ sd:element ref="Name"/>

<xsd:choice>

<xsd:element ref="Units"/>

<xsd:element ref="Unitless"/>

$</ x s d$ :choice $>$

$<x$ d:element ref="Notes" minOccurs="0"/>

$<$ xsd:sequence $>$

<xsd:attribute name="id" type="xsd:ID" use="required"/>

<xsd:attribute name="type" type="xsd:string" use="optional"/>

$<$ xsd:complexType>

$<$ xsd:element>

<xsd:element name="MeasurementTechniqueDetails" minOccurs="0" maxOccurs="unbounded">

<xsd:annotation>

<xsd:documentation >

MeasurementTechniqueDetails

This element declares the content model for MeasurementTechniqueDetails, which contains a description of a measurement technique referenced by the PropertyData element. MeasurementTechniqueDetails has one required attribute, id, which may be arbitranily assigned but must be unique among id attributes assigned elsewhere in a MatML document.

MeasurementTechniqueDetails is composed of the following elements.

Name contains the name of the measurement technique and has one optional attribute, authority, for identifying an authoritative source of measurement techniques. Name must occur once and only once within the MeasurementTechniqueDetails element.

Notes contains any additional information concerning the measurement technique, such as a description of the technique, and may occur once or not at all within the MeasurementTechniqueDetails element.

$<$ xsd:documentation $>$

$<$ xsd:annotation $>$

<xsd:complexType>

<xsd:sequence>

$<x$ sd:element ref="Name"/>

$<x s d: e l e m e n t$ ref="Notes" minOccurs="0"/>

$<$ xsd:sequence $>$

$<x s d: a t t r i b u t e ~ n a m e=" i d "$ type="xsd:ID" use="required"/>

$<$ xsd:complexType $>$

$<$ xsd:element>

<xsd:element name="SpecimenDetails" minOccurs="0" maxOccurs="unbounded"> <xsd:annotation>

<xsd:documentation> 


\section{SpecimenDetails}

This element declares the content model for SpecimenDetails, which contains a description of a specimen referenced by the PropertyData element. SpecimenDetails has one required attribute, id, which may be arbitrarily assigned but must be unique among id attributes assigned elsewhere in a MatML document. SpecimenDetails also has one optional attribute, type, for specifying the type of the specimen (examples include "cylindrical," "rectangular," "full cross-section," "pressed," etc.) SpecimenDetails is composed of the following elements.

Name contains the name of the specimen and has one optional attribute, authority, for identifying an authoritative source of specimen names. Name must occur once and only once within the SpecimenDetails element.

Notes contains any additional information concerning the specimen and may occur once or not at all within the SpecimenDetails element.

$</ x s d$ :documentation $>$

$<$ xsd:annotation $>$

<xsd:complexType>

<xsd:sequence>

$<x$ d:element ref="Name"/>

<xsd:element ref="Notes" minoccurs="0"/>

$<$ xsd:sequence $>$

<xsd:attribute name="id" type="xsd:ID" use="required"/>

$<x$ sd:attribute name="type" type="xsd:string" use="optional"/> $<$ xsd:complexType $>$

$<$ xsd:element>

<xsd:element name="ParameterDetails" minOccurs="0" maxOccurs="unbounded"> $<x s d: a n n o t a t i o n>$

$<$ <sd:documentation $>$

\section{ParameterDetails}

This element declares the content model for ParameterDetails, which contains a description of a parameter referenced by the ParameterValue element. ParameterDetails has one required attribute, id, which may be arbitrarily assigned but must be unique among id attributes assigned elsewhere in a MatML document. ParameterDetails is composed of the following elements.

Name contains the name of the parameter and has one optional attribute, authority, for identifying an authoritative source of parameter names. Name must occur once and only once within the ParameterDetails element.

Units and Unitless are mutually exclusive elements for describing the parameter's units. Units or Unitless must occur once and only once within the ParameterDetails element. For additional information, see the documentation for the Units and Unitless elements.

Notes contains any additional information concerning the parameter and may occur once or not at all within the ParameterDetails element.

$<$ xsd:documentation $>$

$<$ xsd:annotation $>$

<xsd:complexType>

<xsd:sequence>

<xsd:element ref="Name"/>

$<x s d$ :choice $>$

<xsd:element ref="Units"/>

<xsd:element ref="Unitless"/>

$<$ xsd:choices

$<x s d:$ element ref="Notes" minOccurs $=" 0 " />$ 


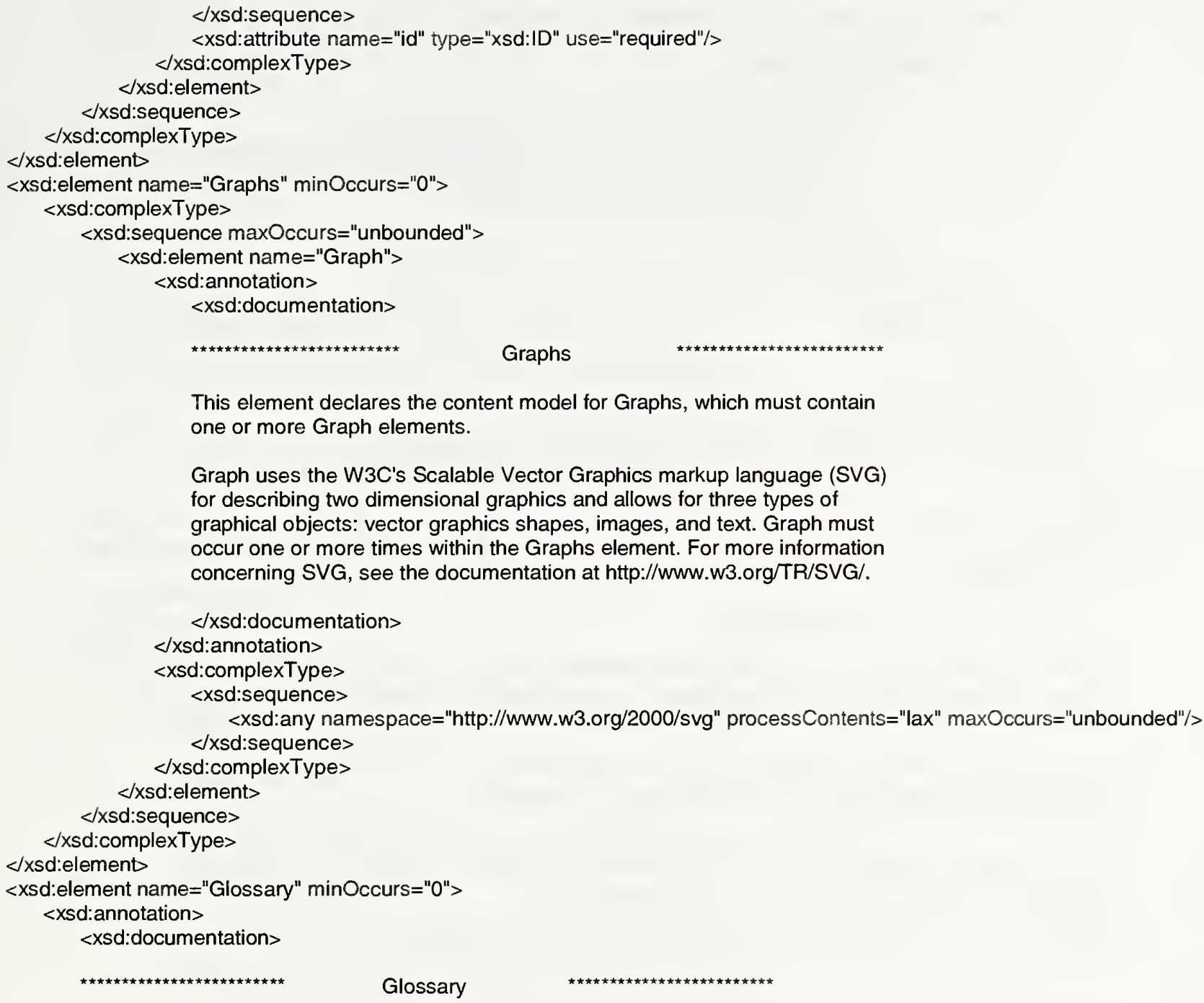

This element declares the content model for Graphs, which must contain one or more Graph elements.

Graph uses the W3C's Scalable Vector Graphics markup language (SVG) for describing two dimensional graphics and allows for three types of graphical objects: vector graphics shapes, images, and text. Graph must occur one or more times within the Graphs element. For more information concerning SVG, see the documentation at http://www.w3.org/TR/SVG/.

$</ x s d:$ documentation $>$

$</ x s d: a n n o t a t i o n>$ <xsd:complexType>

Glossary

This element declares the content model for Glossary, which contains descriptions of material and property terms used in the document. Glossary must contain one or more Term elements each of which is composed of the following elements.

Name contains the term's name and has one optional attribute, authority, for identifying an authoritative source of terms. Name must occur once and only once within the Term element.

Definition contains the term's definition and must occur once and only once within the Term element.

Abbreviation contains the term's abbreviations and may occur zero or more times within the Term element.

Synonym contains the term's synonyms and may occur zero or more times within the Term element.

Notes contains any additional information concerning the term and may occur once or not at all within the Term element.

$<$ xsd:documentation $>$

$<$ xsd:annotation>

<xsd:complexType> 
$<$ xsd:sequence maxOccurs="unbounded" $>$

<xsd:element name="Term">

<xsd:complexType>

<xsd:sequence>

$<x$ s:element ref="Name"/>

$<x$ sd:element name="Definition" type="xsd:string"/>

<xsd:element name="Abbreviation" type="xsd:string" minOccurs="0" maxOccurs="unbounded"/> <xsd:element name="Synonym" type="xsd:string" minOccurs="0" maxOccurs="unbounded"/> $<x$ sd:element ref="Notes" minOccurs="0"/>

$</ x$ sd:sequence>

$<$ xsd:complexType $>$

$<$ xsd:element>

$</ x s d$ :sequence $>$

$<$ ixsd:complexType>

$</ x$ sd:element $>$

$</ x s d$ :sequence $>$

<xsd:attribute name="id" type="xsd:ID" use="optional"/>

<xsd:attribute name="layers" type="xsd:integer" use="optional"/>

<xsd:attribute name="local_frame_of_reference" type="xsd:ID" use="optional"/>

$</ x s d$ :complexType>

$</ x$ sd:element $>$

$<$ xsd:element name="Characterization">

<xsd:annotation>

<xsd:documentation>

\section{Characterization}

This element declares the content model for Characterization, which contains a description of the chemical composition of the bulk material or component and is composed of the following elements.

Formula contains a string representation of the chemical formula for the bulk material or component and must occur once and only once within the Characterization element.

ChemicalComposition contains a description of the compounds and elements that comprise the bulk material or component and may occur once or not at all within the Characterization element. For additional information, see the documentation for the ChemicalComposition element.

PhaseComposition contains a description of the phases that comprise the bulk material or component and may occur zero or more times within the Characterization element. For additional information, see the documentation for the PhaseComposition element.

DimensionalDetails contains information relating to component or bulk material dimensional characteristics such as grain size, porosity, precipitate size and distribution, etc., and may occur zero or more times within the Characterization element. For additional information, see the documentation for the DimensionalDetails element.

Notes contains any additional information concerning the Characterization and may occur once or not at all within the Characterization element.

$</$ xsd:documentation $>$

$</ x s d: a n n o t a t i o n>$

<xsd:complexType>

$<x s d$ :sequence>

<xsd:element name="Formula" type="xsd:string" >

$<$ xsd:annotation >

$<$ xsd:documentation $>$

Formula

This element declares the content model for Formula, which contains a 
stning representation of the chemical formula for the bulk material or component.

$<$ xsd:documentation $>$

$<$ xsd:annotation>

$<$ xsd:element

<xsd:element name="ChemicalComposition" minOccurs="0">

$<$ <sd:annotation >

<xsd:documentation>

\section{ChemicalComposition}

This element declares the content model for ChemicalComposition, which contains a detailed description of the compounds and elements that comprise the bulk material or component. ChemicalComposition must contain at least one Compound element or Element element but may contain as many of each element as needed.

Compound contains a description of a compound. For additional information, see the documentation for the Compound element.

Element contains a description of an element. For additional information, see the documentation for the Element element.

$</ x$ sd:documentation $>$

$</ x$ sd:annotation $>$

<xsd:complexType>

<xsd:choice maxOccurs="unbounded">

$<x s d: e l e m e n t$ name $=$ "Compound" $>$

$<x$ sd:annotation>

<xsd:documentation>

Compound

This element declares the content model for Compound, which contains the elemental description of a chemical compound and is composed the following elements.

Element contains the description of a chemical element and must occur one or more times within the Compound element. For additional information, see the documentation for the Element element.

Concentration contains the concentration of the compound and may occur once or not at all within the Compound element. For additional information, see the documentation for the Concentration element.

Notes contains any additional information concerning the compound and may occur once or not at all within the Compound element.

$</ x s d$ documentation $>$

$<$ xsd:annotation>

<xsd:complexType>

$<x s d$ :sequence>

<xsd:element ref="Element" maxOccurs="unbounded"/>

$<x$ sd:element ref="Concentration" minOccurs="0"/>

$<x s d: e l e m e n t$ ref="Notes" minOccurs="0"/>

$</$ xsd:sequence $>$

$<$ xsd:complexType>

$<$ xsd:element>

<xsd:element ref="Element"/>

$<$ xsd:choices

$<$ xsd:complexType>

$<$ xsd:element

<xsd:element name="PhaseComposition" minOccurs="0" maxOccurs="unbounded">

$<x s d: a n n o t a t i o n>$

$<$ xsd:documentation $>$ 
This element declares the content model for PhaseComposition, which contains a description of a phase that comprises the bulk material or component and is composed of the following elements.

Name contains the name of the phase and has one optional attribute, authority, for identifying an authoritative source of phase names. Name must occur once and only once within the PhaseComposition element.

Concentration contains the concentration of the phase and may occur once or not at all within the PhaseComposition element. For additional information, see the documentation for the Concentration element.

PropertyData contains property data for the phase and may occur zero or more times within the PhaseComposition element. For additional information, see the documentation for the PropertyData element.

Notes contains any additional information concerning the phase and may occur once or not at all within the PhaseComposition element.

$<$ xsd:documentation $>$

$</ x$ sd:annotation $>$

<xsd:complexType>

<xsd:sequence>

<xsd:element ref="Name"/>

$<x$ sd:element ref="Concentration" minOccurs="0"/>

$<x$ sd:element ref="PropertyData" minoccurs="0" maxOccurs="unbounded"/>

$<x s d:$ element ref="Notes" minoccurs="0"/>

$<$ xsd:sequence>

$<$ xsd:complexType>

$<$ xsd:element

$<x$ sd:element name="DimensionalDetails" minOccurs="0" maxOccurs="unbounded">

$<$ xsd:annotation $>$

$<$ xsd:documentation $>$

\section{DimensionalDetails}

This element declares the content model for DimensionalDetails, which contains a description of a dimensional characteristic (e.g. grain size, porosity, precipitate size and distribution, etc.) of the bulk material or component and is composed of the following elements.

Name contains the name of the characteristic and has one optional attribute, authority, for identifying an authoritative source of dimensional characteristic names. Name must occur once and only once within the DimensionalDetails element.

Value contains the value of the dimensional characteristic and has one required attribute, format, for indicating the format of the value ("float," "integer," "string," or "exponential") found in Value. Value must occur once and only once within the DimensionalDetails element.

Units contains the units for the value of the dimensional characteristic and must occur once and only once within the DimensionalDetails element. For additional information, see the documentation for the Units element.

Qualifier contains any qualifier pertinent to the value of the dimensional characteristic (e.g. "min," "max," etc.) and may occur once or not at all within the DimensionalDetails element.

Notes contains any additional information concerning the dimensional characteristic and may occur once or not at all within the DimensionalDetails element. 
xsd:documentation>

$<$ xsd:annotation>

<xsd:complexType>

$<x$ sd:sequence>

<xsd:element ref="Name"/>

$<x$ sd:element ref $=$ "Value"/>

$<x$ sd:element ref="Units"/>

$<x s d:$ element ref="Qualifier" minoccurs="0"/>

$<x$ sd:element ref="Notes" minOccurs="0"/>

$</$ xsd:sequence $>$

$<$ xsd:complexType>

$<$ xsd:elements

$<x$ d:element ref="Notes" minoccurs="0"/>

$<$ xsd:sequence $>$

$<$ xsd:complexType $>$

$<$ /xsd:element $>$

<xsd:element name="Class" type="xsd:string">

<xsd:annotation>

<xsd:documentation>

Class

This element declares the content model for Class, which contains a string representing the class of the bulk material or component.

$<$ xsd:documentation $>$

$</$ xsd:annotation $>$

$<$ xsd:element $>$

<xsd:element name="ComponentDetails">

$<x s d$ :annotation>

<xsd:documentation>

\section{ComponentDetails}

This element declares the content model for ComponentDetails, which contains a description of a component within the bulk material and has one optional attribute, id, which may be used as an identification specifier for the component and is especially useful for complex systems such as composite laminates.

ComponentDetails is composed of the following elements.

Name contains the component's name and has one optional attribute, authority, for identifying an authoritative source of component names. Name must occur once and only once within the ComponentDetails element.

Class contains the component's class and may occur once or not at all within the ComponentDetails element.

Subclass contains the component's subclass(es) and may occur zero or more times within the ComponentDetails element.

Specification contains the component's specification(s) and has one optional attribute, authority, for identifying an authoritative source of component specifications. Specification may occur zero or more times within the ComponentDetails element.

Source contains the name of the source of the component and may occur once or not at all within the ComponentDetails element.

Form contains the form of the component and may occur once or not at all within the ComponentDetails element.

ProcessingDetails contains a description of a processing step for 
the component and may occur zero or more times within the ComponentDetails element. For additional information, see the documentation for the ProcessingDetails element.

Geometry contains a description of the geometry of the component and may occur once or not at all within the ComponentDetails element For additional information, see the documentation for the Geometry element.

Characterization contains the characterization of the component, including the formula, chemical composition, phase composition, and dimensional details. Characterization may occur once or not at all within the ComponentDetails element. For additional information, see the documentation for the Characterization element.

PropertyData contains the property data for the component and may occur zero or more times within the ComponentDetails element. For additional information, see the documentation for the PropertyData element.

AssociationDetails contains a description of a relationship of the component to another component and may occur zero or more times within the ComponentDetails element. For additional information, see the documentation for the AssociationDetails element.

Notes contains any additional information concerning the component and may occur once or not at all within the ComponentDetails element.

ComponentDetails contains a description of a component within the component and is used to support encoding of information for complex materials systems such as composites. ComponentDetails may occur zero or more times within the ComponentDetails element.

$</ x$ sd:documentation $>$

$<$ xsd:annotation $>$

<xsd:complexType>

<xsd:sequence>

$<x$ sd:element ref="Name"/>

$<x$ sd:element ref="Class" minOccurs="0"/>

<xsd:element ref="Subclass" minOccurs="0" maxOccurs="unbounded"/>

$<x s d: e l e m e n t$ ref="Specification" minOccurs="0" maxOccurs="unbounded"/>

$<x$ sd:element ref="Source" minOccurs="0"/>

$<x$ d:element ref="Form" minOccurs="0"/>

<xsd:element ref="ProcessingDetails" minOccurs="0" maxOccurs="unbounded"/>

<xsd:element ref="Geometry" minOccurs="0"/>

$<x s d: e l e m e n t$ ref $=$ "Characterization" minoccurs $=" 0 " />$

<xsd:element ref="PropertyData" minOccurs="0" maxOccurs="unbounded"/>

<xsd:element name="AssociationDetails" minOccurs="0" maxOccurs="unbounded">

<xsd:annotation>

<xsd:documentation>

\section{AssociationDetails}

This element declares the content model for AssociationDetails, which contains a description of a relationship of the component to another component in a complex material system such as a composite, weld, or multilayer material. AssociationDetails is composed of the following elements.

Associate contains the name of a component's associate. For example, a TiC coating has been placed on AISI 1018 steel coupons. The Associate of the steel, then, is the "titanium carbide coating." Associate must occur once and only once within the AssociationDetails element.

Relationship contains a description of the relationship between a component and the associate. For example, the associate of the "steel" 
component is the "titanium carbide coating." The relationship of the "steel" to the "titanium carbide coating" is that the steel is the "substrate" for the coating. Relationship must occur once and only once within the AssociationDetails element.

Notes contains any additional information concerning the association and may occur once or not at all within the AssociationDetails element.

$<$ xsd:documentation $>$

$</ x s d: a n n o t a t i o n>$

$<x$ d:complexType>

$<x$ d:sequence $>$

<xsd:element name="Associate" type="xsd:string">

<xsd:annotation>

<xsd:documentation>

\section{Associate}

This element declares the content model for Associate, which contains a string representing a component's associate.

$</$ xsd:documentation $>$ $<$ xsd:annotation $>$

$<$ xsd:element

$<x s d: e l e m e n t$ name="Relationship" type="xsd:string"> $<x$ sd:annotation $>$

<xsd:documentation>

Relationship

This element declares the content model for Relationship, which contains a string representing the relationship between a component and the associate.

$</ x s d$ :documentation $>$ $<$ xsd:annotation>

$<$ xsd:element

<xsd:element ref="Notes" minOccurs="0"/> $<$ xsd:sequence $>$

$<$ xsd:complexType>

$<$ xsd:element

$<x s d: e l e m e n t$ ref="Notes" minOccurs="0"/>

$<x$ sd:element ref="ComponentDetails" minOccurs="0" maxOccurs="unbounded"/>

$<$ xsd:sequence $>$

<xsd:attribute name="id" type="xsd:ID" use="optional"/>

$<$ xsd:complexType>

$<$ ixsd:element>

$<x s d: e l e m e n t$ name="Concentration">

$<$ xsd:annotation>

<xsd:documentation>

\section{Concentration}

This element declares the content model for Concentration and is composed of the following elements.

Value contains the value of the concentration and has one required attribute, format, for indicating the format of the value ("float," "integer," "string," or "exponential") found in Value. Value must occur once and only once within the Concentration element.

Units contains the units for the value of the concentration and must occur once and only once within the Concentration element. For additional information, see the documentation for the Units element.

Qualifier contains any qualifier pertinent to the value of the concentration 
(e.g. "min," "max," etc.) and may occur once or not at all within the Concentration element.

Notes contains any additional information concerning the concentration and may occur once or not at all within the Concentration element.

$<$ xsd:documentation $>$

$<$ xsd:annotation $>$

<xsd:complexType>

$<x s d$ :sequence $>$

<xsd:element ref="Value"/>

$<x s d: e l e m e n t$ ref="Units"/>

<xsd:element ref="Qualifier" minOccurs="0"/>

$<x s d: e l e m e n t$ ref="Notes" minOccurs="0"/>

$<$ ixsd:sequence $>$

$<$ xsd:complexType>

$<$ xsd:element>

$<x$ sd:element name="Element" $>$

$<x s d$ :annotation>

$<x s d$ documentation>

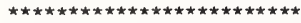

Element

This element declares the content model for Element and is composed of the following elements.

Symbol contains the symbol for the chemical element, which is one among those enumerated by the ChemicalElementSymbol datatype. Symbol has one optional attribute, subscript, for indicating the subscript (formula units) of the chemical element. Symbol must occur once and only once within the Element element. For additional information, see the documentation for the ChemicalElementSymbol datatype.

Concentration contains the concentration of the element and may occur once or not at all within the Element element. For additional information, see the documentation for the Concentration element.

Notes contains any additional information concerning the element and may occur once or not at all within the Element element.

$<$ xsd:documentation >

$<$ xsd:annotation $>$

<xsd:complexType>

$<x s d$ :sequence $>$

$<x$ sd:element name $=$ "Symbol">

$<x s d: a n n o t a t i o n>$

<xsd:documentation>

Symbol

This element declares the content model for Symbol, which contains the symbol for the chemical element. The entry for Symbol is selected from among the strings enumerated by the ChemicalElementSymbol datatype. Symbol has one optional attribute, subscript, for indicating the subscript (formula units) of the chemical element.

$</ x s d:$ documentation $>$

$<$ xsd:annotation $>$

<xsd:complexType>

<xsd:simpleContent

<xsd:extension base="ChemicalElementSymbol">

<xsd:attribute name="subscript" type="xsd:string" use="optional" default=" 1 "/> $<$ xsd:extension>

$<$ xsd:simpleContent $>$

$<$ xsd:complexType> 


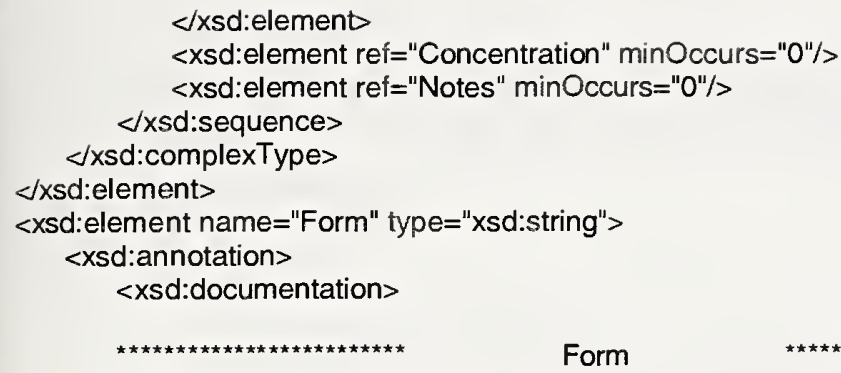

This element declares the content model for Form, which contains a string representing the form of the bulk material or component.

$</ x s d$ documentation $>$

$<\mathrm{xsd}$ :annotation >

$<$ xsd:element>

<xsd:element name="Geometry">

<xsd:annotation>

$<$ <sd:documentation>

\section{Geometry}

This element declares the content model for Geometry, which contains a description of the geometry of the bulk material or component and is composed of the following elements.

Shape contains a description of the shape of the bulk material or component and must occur once and only once within the Geometry element.

Dimensions contains the dimensions of the bulk material or component and may occur once or not at all within the Geometry element.

Orientation contains the orientation of the bulk material or component and may occur once or not at all within the Geometry element.

Notes contains any additional information concerning the geometry and may occur once or not at all within the Geometry element.

$</$ xsd:documentation $>$

$<$ xsd:annotation>

<xsd:complexType>

$<x$ sd:sequences

<xsd:element name="Shape" type="xsd:string">

$<x s d: a n n o t a t i o n>$

<xsd:documentation>

\section{Shape}

This element declares the content model for Shape, which contains a string representing the shape of the bulk material or component.

$<$ xsd:documentation $>$

$<$ xsd:annotation $>$

$</$ xsd:element

<xsd:element name="Dimensions" type="xsd:string" minOccurs="0">

<xsd:annotation>

$<$ <sd:documentation >

\section{Dimensions}

This element declares the content model for Dimensions, which contains a string representing the dimensions of the bulk material or component. 


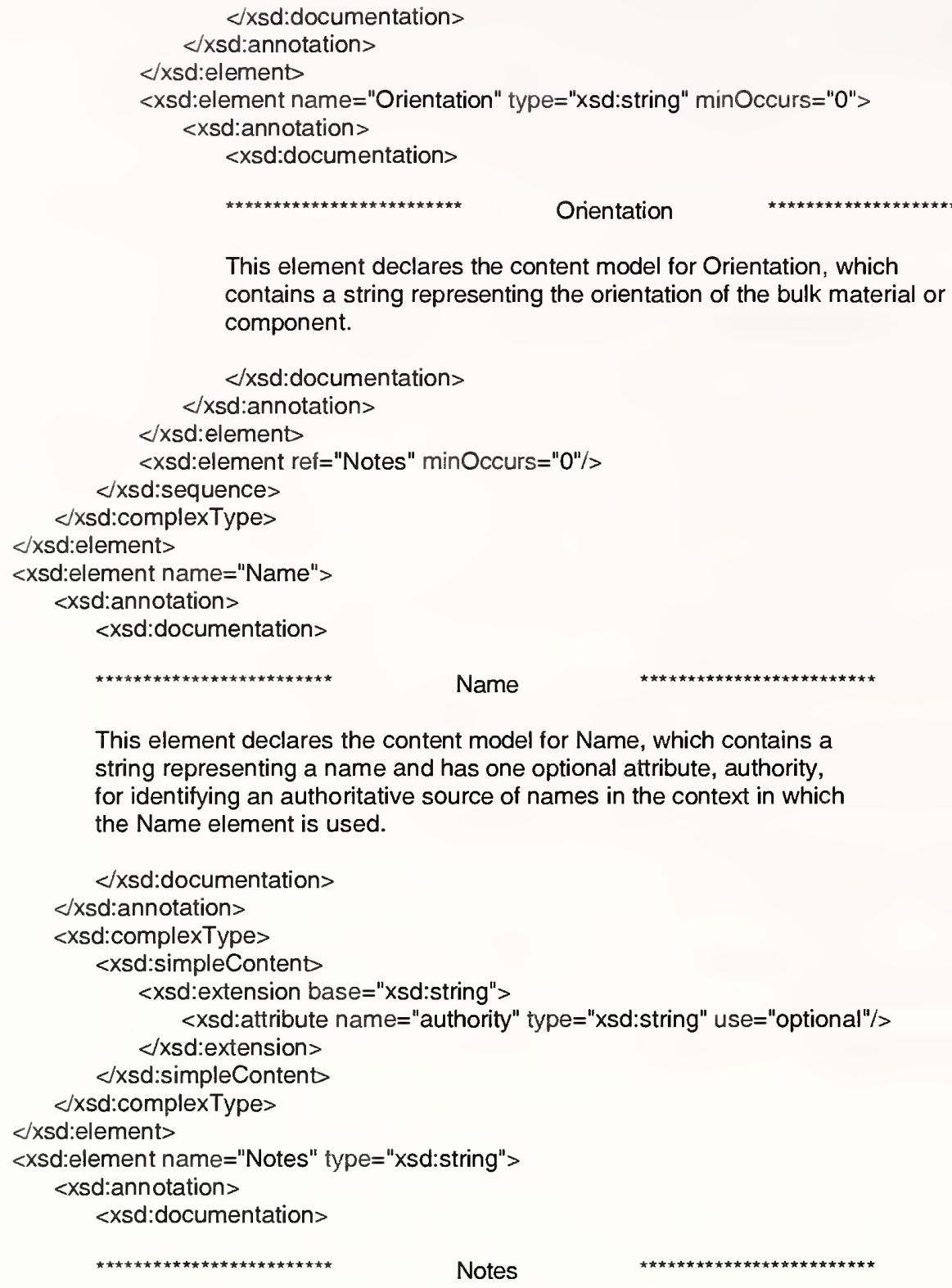

This element declares the content model for Orientation, which contains a string representing the orientation of the bulk material or component.

$</ x s d$ documentation $>$

$</ x s d: a n n o t a t i o n>$

$</$ xsd:element

$<x s d:$ element ref="Notes" minOccurs="0"/>

$\langle$ xsd:sequence $>$

$<$ xsd:complexType>

$\langle$ xsd:element $>$

<xsd:element name $="$ Name" $>$

<xsd:annotation>

$<$ <sd:documentation>

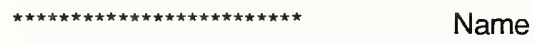

This element declares the content model for Name, which contains a string representing a name and has one optional attribute, authority, for identifying an authoritative source of names in the context in which the Name element is used.

$</ x s d: d o c u m e n t a t i o n>$

$<$ xsd:annotation>

<xsd:complexType>

$<x$ sd:simpleContent <xsd:extension base="xsd:string">

<xsd:attribute name="authority" type="xsd:string" use="optional"/> $<$ xsd:extension>

$<$ <sd:simpleContent

$<$ xsd:complexType>

$<$ <sd:element>

$<x$ sd:element name="Notes" type="xsd:string">

<xsd:annotation >

<xsd:documentation>

Notes

This element declares the content model for Notes, which contains a string representing descriptive notes.

$<$ xsd:documentation $>$

$<$ xsd:annotation>

$<$ xsd:element $>$

<xsd:element name="ParameterValue">

<xsd:annotation>

<xsd:documentation>

\section{ParameterValue}

This element declares the content model for ParameterValue, which contains the value of a parameter. ParameterValue has two required attributes.

The first attribute, parameter, references an id attribute specified in a ParameterDetails element so that the descriptive details of the parameter 
are tied to the value.

The second attribute, format, indicates the format of the value ("float,"

"integer," "string," or "exponential") found in ParameterValue.

$<$ xsd:documentation $>$

$<$ /xsd:annotation>

<xsd:complexType>

<xsd:simpleContent $>$

<xsd:extension base="xsd:string">

<xsd:attribute name="parameter" type="xsd:IDREF" use="required"/>

<xsd:attribute name="format" use="required"> <xsd:simpleType>

<xsd:restriction base="xsd:string">

$<x$ sd:enumeration value $=$ "float"/>

$<x$ sd:enumeration value $=$ "integer"/>

$<$ xsd:enumeration value $=$ "string"/>

$<x$ sd:enumeration value="exponential"/> $<$ /xsd:restriction>

$<$ xsd:simpleType $>$

$</$ xsd:attribute $>$

$<$ xsd:extension $>$

$<$ xsd:simpleContent $>$

$<$ xsd:complexType $>$

$<$ <xsd:element>

$<x$ sd:element name $=$ "ProcessingDetails">

<xsd:annotation>

<xsd:documentation>

\section{ProcessingDetails}

This element declares the content model for ProcessingDetails, which contains a description of a processing step for the bulk material or component. ProcessingDetails is composed of the following elements.

Name contains the name of the processing step and has one optional attribute, authority, for identifying an authoritative source of processing step names. Name must occur once and only once within the ProcessingDetails element.

ParameterValue contains the value of a parameter under which the processing step occurred and may occur zero or more times within the ProcessingDetails element. For additional information, see the documentation for the ParameterValue element.

Result contains a description of the outcome or result of the processing step and may occur once or not at all within the ProcessingDetails element.

Notes contains any additional information concerning the processing step and may occur once or not at all within the ProcessingDetails element.

$</$ xsd:documentation $>$

$</$ xsd:annotation $>$

$<x$ sd:complexType>

$<x$ sd:sequence

$<$ xsd:element ref="Name"/>

<xsd:element ref="ParameterValue" minOccurs="0" maxOccurs="unbounded"/>

<xsd:element name="Result" type="xsd:string" minOccurs="0">

$<$ xsd:annotation>

<xsd:documentation>

Result

This element declares the content model for Result, which contains a 
string representing the result of a processing step.

$</ x$ sd:documentation $>$

$<$ xsd:annotation>

$<$ xsd:element

<xsd:element ref="Notes" minOccurs="0"/>

$<$ xsd:sequence>

$<$ xsd:complexType>

$</ x$ sd:element $>$

$<$ xsd:element name="PropertyData">

<xsd:annotation>

<xsd:documentation>

$$
\text { PropertyData }
$$

This element declares the content model for PropertyData, which contains property data. PropertyData has four attributes.

The first attribute, property, is required and references an id attribute specified in a PropertyDetails element so that the descriptive details for the property are tied to the data found in the Data element.

The second attribute, technique, is optional and references an id attribute Specified in a MeasurementTechniqueDetails element so that the Descriptive details for the measurement technique are tied to the data found In the Data element.

The third attribute, source, is optional and references an id attribute specified in a DataSourceDetails element so that the descriptive details for the data source are tied to the data found in the Data element.

The fourth attribute, specimen, is optional and references an id attribute specified in a SpecimenDetails element so that the descriptive details for the specimen are tied to the data found in the Data element.

PropertyData is composed of the following elements.

Data contains the property data and has one required attribute, format, for indicating the format of the data ("float," "integer," "string," or "exponential") found in Data. Data must occur once and only once within the PropertyData element.

Qualifier contains any qualifier(s) pertinent to the data in Data (e.g. "min," "max," etc.) and may occur once or not at all within the PropertyData element.

Uncertainty contains the measurement uncertainty(ies) of the data in Data and may occur once or not at all within the PropertyData element. For additional information, see the documentation for the Uncertainty element.

ParameterValue contains the value(s) of a parameter under which the data were determined and may occur zero or more times within the PropertyData element. For additional information, see the documentation for the ParameterValue element.

Notes contains any additional information concerning the property data and may occur once or not at all within the PropertyData element.

Note - Multiple entries in the Data, Qualifier, Uncertainty Value, and ParameterValue elements must be comma delimited and synchronized across elements, i.e., the number of entries in each of these four elements must be equal.

$</ x s d$ :documentation $>$

$<$ xsd:annotation> 
<xsd:complexType>

<xsd:sequence>

<xsd:element name="Data">

<xsd:annotation>

<xsd:documentation>

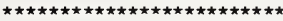

Data

This element declares the content model for Data, which contains property data and has one required attribute, format, for indicating the format of the data ("float," "integer," "string," or "exponential") found in Data.

$</ x s d:$ documentation $>$

$</ x$ sd:annotation>

<xsd:complexType>

$<x s d:$ simpleContent

<xsd:extension base="xsd:string">

<xsd:attribute name="format" use="required"> <xsd:simpleType>

$<x s d$ :restriction base="xsd:string">

$<x$ sd:enumeration value $=$ "float" $/>$

<xsd:enumeration value="integer"/>

$<x$ sd:enumeration value $=$ "string"/>

$<x$ d:enumeration value $=$ "exponential"/> $</ x s d$ :restriction $>$

$<$ xsd:simpleType $>$

$<$ xsd:attribute>

$<$ xsd:extension>

$<$ xsd:simpleContent>

$<$ xsd:complexTypes

$<$ xsd:element

<xsd:element ref="Qualifier" minOccurs="0"/>

<xsd:element name="Uncertainty" minOccurs="0">

$<x$ sd:annotation >

<xsd:documentation>

\section{Uncertainty}

This element declares the content model for Uncertainty, which contains a description of the measurement uncertainty of the data. Uncertainty is composed of the following elements.

Value contains the value of the uncertainty and has one required attribute, format, for indicating the format of the value ("float," "integer," "string," or "exponential") found in Value. Value must occur once and only once within the Uncertainty element.

Units contains the units for the value of the uncertainty and must occur once and only once within the Uncertainty element. For additional information, see the documentation for the Units element.

Notes contains any additional information concerning the uncertainty, such as a description of the evaluation of the uncertainty, and may occur once or not at all within the Uncertainty element.

$<$ xsd:documentation>

$<$ xsd:annotation $>$

<xsd:complexType>

$<x$ sd:sequence $>$

$<x$ sd:element ref="Value"/>

$<x$ sd:choice>

$<x$ sd:element ref="Units"/>

$<x$ sd:element ref="Unitless"/>

$<$ xsd:choice>

$<x s d: e l e m e n t$ ref="Notes" minOccurs="0"/> 


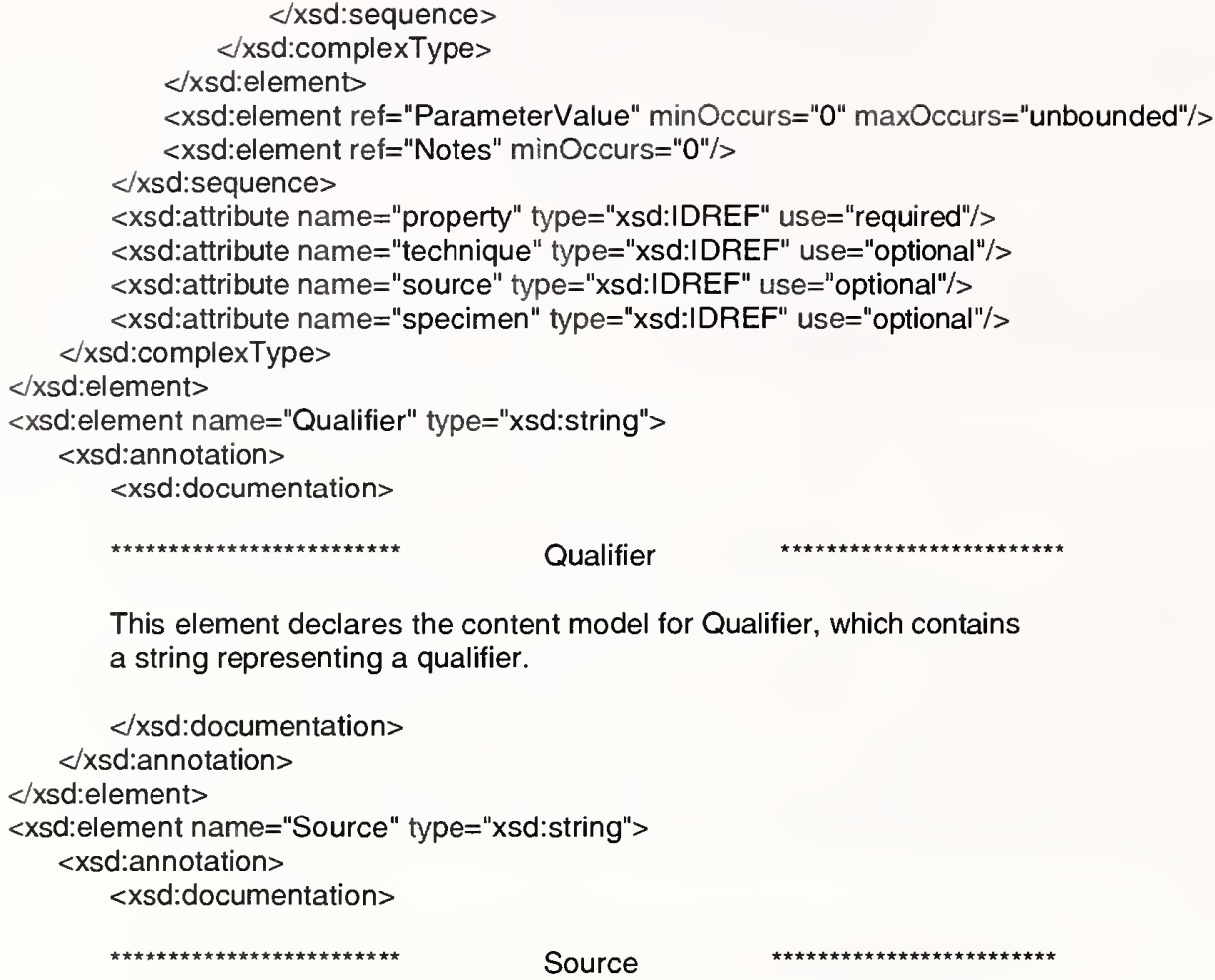

This element declares the content model for Qualifier, which contains a string representing a qualifier.

$<$ xsd:documentation>

$<$ xsd:annotation>

$<$ xsd:element $>$

<xsd:element name="Source" type="xsd:string">

<xsd:annotation>

$<$ <sd:documentation>

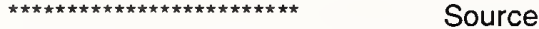

This element declares the content model for Source, which contains a string representing the source of the bulk material or component.

$</$ xsd:documentation $>$

$<$ xsd:annotation $>$

$<$ xsd:element>

$<x$ sd:element name="Specification">

$<x$ sd:annotation>

$<x$ sd:documentation>

\section{Specification}

This element declares the content model for Specification, which contains a string representing the specification for the bulk material or component and has one optional attribute, authority, for identifying an authoritative source of specifications.

$</$ xsd:documentation $>$

$<$ xsd:annotation $>$

<xsd:complexType>

$<x$ sd:simpleContent

<xsd:extension base="xsd:string">

<xsd:attribute name="authority" type="xsd:string" use="optional"/> $<$ xsd:extension $>$

$<$ xsd:simpleContent

$<$ xsd:complexType $>$

$<$ xsd:element $>$

<xsd:element name="Subclass" type="xsd:string">

$<x s d: a n n o t a t i o n>$

<xsd:documentation>

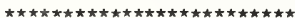

\section{Subclass}

This element declares the content model for Subclass, which contains a string representing the subclass of the bulk material or component. 
$<$ xsd:documentation>

$<$ xsd:annotation $>$

$\langle$ xsd:element $>$

$<x$ sd:element name $="$ Unit">

$<x s d: a n n o t a t i o n>$

$<x$ sd:documentation >

Unit

This element declares the content model for Unit, which contains a unit and has two optional attributes.

The first attribute, power, is used to indicate the exponent for Unit.

The second attribute, description, is used to describe Unit.

Note - Multiple Unit elements are multiplied together to form units. Division is specified by setting the power attribute of Unit equal to "-1." For additional information, see the documentation for the Units element.

$<$ xsd:documentation $>$

$\checkmark$ xsd:annotations

<xsd:complexType>

$<x$ sd:simpleContents

$<x$ sd:extension base="xsd:string">

<xsd:attribute name="power" type="xsd:decimal"/>

<xsd:attribute name="description" type="xsd:string"/>

$<$ xsd:extension>

$<$ xsd:simpleContent

$\langle$ xsd:complexType

$<$ xsd:element $>$

$<x$ d:element name="Unitless">

$<x s d: a n n o t a t i o n>$

<xsd:documentation>

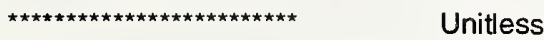

This element declares the content model for Unitless, which is an empty element used whenever a property, parameter, or uncertainty value has no units.

$</$ xsd:documentation $>$

$<$ xsd:annotation >

<xsd:complexType/>

$\langle$ xsd:element>

<xsd:element name="Units">

$<x$ sd:annotation>

<xsd:documentation>

\section{Units}

This element declares the content model for Units, which contains units and has four optional attributes.

The first attribute, system, is used to indicate the units system, such as "SI."

The second attribute, factor, is used to indicate a constant multiplier in floating point format.

The third attribute, name, is used to indicate the name of the units

The fourth attribute, description, is used to describe the units.

Units is composed of the following elements. 
Unit contains a unit and must occur one or more times within the Units element. For additional information, see the documentation for the Unit element.

Note - Multiple Unit elements are multiplied together to form the units. Division is specified by using setting the power attribute of Unit equal to "-1."

$</ x s d$ documentation $>$

$<$ xsd:annotation>

<xsd:complexType>

$<x$ sd:sequence $>$

<xsd:element ref="Unit" maxOccurs="unbounded"/>

$<$ xsd:sequence $>$

<xsd:attribute name="system" type="xsd:string"/>

<xsd:attribute name="factor" type="xsd:float"/>

$<x s d$ :attribute name="name" type="xsd:string"/>

<xsd:attribute name="description" type="xsd:string"/>

$</ x$ sd:complexType $>$

$<$ xsd:element>

<xsd:element name="Value">

$<$ <sd:annotation>

<xsd:documentation>

Value

This element declares the content model for value, which contains a string representing a value. Value has one required attribute, format, for indicating the format of the value ("float," "integer," "string," or "exponential") found in Value.

$<$ xsd:documentation $>$

$<$ xsd:annotation>

<xsd:complexType>

<xsd:simpleContent>

<xsd:extension base="xsd:string">

<xsd:attribute name="format" use="required"> <xsd:simpleType>

<xsd:restriction base="xsd:string">

$<x$ sd:enumeration value $=$ "float" $/>$

$<x$ sd:enumeration value="integer"/>

$<$ xsd:enumeration value="string"/>

$<$ xsd:enumeration value $=$ "exponential"/>

$</$ xsd:restriction $>$

$<$ xsd:simpleType $>$

$</$ xsd:attribute $>$

$<$ xsd:extension>

$<$ xsd:simpleContent

$<$ xsd:complexType $>$

$<$ xsd:element $>$

<xsd:simpleType name="ChemicalElementSymbol">

<xsd:annotation>

<xsd:documentation>

\section{ChemicalElementSymbol}

This datatype enumerates the valid strings representing chemical elements, which may be used in the Symbol element.

$<$ xsd:documentation>

$<$ xsd:annotation $>$

<xsd:restriction base="xsd:string">

$<$ xsd:enumeration value $=" \mathrm{H} " />$

$<$ xsd:enumeration value $=" \mathrm{He} " />$

$<x$ sd:enumeration value $=" \mathrm{Li} " />$ 
$<x$ sd:enumeration value $=" \mathrm{Be}$ "/ $/>$ $<x$ sd:enumeration value $=" \mathrm{~B}^{\prime \prime} />$ <xsd:enumeration value $=" \mathrm{C} " />$ $<x$ sd:enumeration value $=" \mathrm{~N} />$ $<x$ sd:enumeration value $=" \mathrm{O} />>$ $<x$ sd:enumeration value $=" F " />$ $<x$ sd:enumeration value $=" \mathrm{Ne}$ "/> $<x$ sd:enumeration value $=" \mathrm{Na} " />$ <xsd:enumeration value="Mg"/> <xsd:enumeration value="Al"/> $<x s d$ :enumeration value $=" \mathrm{Si} " />$ $<x$ sd:enumeration value $=" \mathrm{P} />$ <xsd:enumeration value="S"/> <xsd:enumeration value $=" \mathrm{Cl} / 1 />$ <xsd:enumeration value $=" \mathrm{Ar} " />$ $<x$ sd:enumeration value $=" \mathrm{~K} " />$ <xsd:enumeration value $=" \mathrm{Ca} " />$ <xsd:enumeration value="Sc"/> $<x$ sd:enumeration value $=" \mathrm{Ti} " />$ $<x$ sd:enumeration value $=" V " />$ <xsd:enumeration value $=" \mathrm{Cr} " />$ <xsd:enumeration value $=" \mathrm{Mn} " />$ <xsd:enumeration value="Fe"/> $<x$ sd:enumeration value $=" \mathrm{Co} / />$ <xsd:enumeration value $=" \mathrm{Ni} " />$ $<x$ sd:enumeration value $=" \mathrm{Cu} />$ $<x$ d:enumeration value $=" \mathrm{Zn} " />$ <xsd:enumeration value="Ga"/> $<x$ sd:enumeration value $=" \mathrm{Ge} " />$ $<x$ d:enumeration value $=" \mathrm{As}$ " $/>$ <xsd:enumeration value="Se"/> $<x s d: e n u r n e r a t i o n ~ v a l u e=" \mathrm{Br} " />$ <xsd:enumeration value $=" \mathrm{Kr} " />$ $<x$ sd:enumeration value $=" \mathrm{Rb} />$ <xsd:enumeration value=" $\mathrm{Sr} / />$ $<x$ sd:enumeration value $=" Y " />$ <xsd:enumeration value $=" \mathrm{Zr} " />$ $<x$ sd:enumeration value $=" \mathrm{Nb}$ "/> $<x$ sd:enumeration value $=" \mathrm{Mo} " />$ $<x$ sd:enumeration value="Tc" $/>$ <xsd:enumeration value="Ru"/> <xsd:enumeration value $=" R h " />$ $<x$ sd:enumeration value $=" \mathrm{Pd}^{\prime \prime} />$ $<x$ sd:enumeration value=" $\mathrm{Ag}^{\prime \prime} />$ <xsd:enumeration value $=" \mathrm{Cd} " />$ <xsd:enumeration value $==\mid \mathrm{n} " />$ <xsd:enumeration value $=" \mathrm{Sn}^{\prime \prime} />$ <xsd:enumeration value $=" \mathrm{Sb} " />$ <xsd:enumeration value $=" \mathrm{Te}$ "1/> <xsd:enumeration value $=" 1 " />$ $<x$ sd:enumeration value $=" X e " />$ $<x$ sd:enumeration value $=" \mathrm{Cs} " />$ <xsd:enumeration value="Ba"/> $<x$ sd:enumeration value $=" \mathrm{La} " />$ $<x$ sd:enumeration value $=" \mathrm{Ce} " />$ $<x$ sd:enumeration value $=" \mathrm{Pr} " />$ $<x$ sd:enumeration value $=" \mathrm{Nd}$ "/> <xsd:enumeration value =" $\mathrm{Pm} " />$ <xsd:enumeration value $=" \mathrm{Sm} " />$ <xsd:enumeration value="Eu"/> $<x$ d:enumeration value $=" \mathrm{Gd}$ "/> $<x$ sd:enumeration value $=" \mathrm{~Tb} " />$ <xsd:enumeration value $=" \mathrm{Dy} " />$ $<x$ sd:enumeration value $=" H o " />$ <xsd:enumeration value $=" E r " />$ $<x$ sd:enumeration value="Tm"/> 
<xsd:enumeration value $=$ "Yb"/> $<x$ sd:enumeration value $=" L \mathrm{Lu} />$ $<x$ sd:enumeration value $=" \mathrm{Hf} " />$ $<x$ sd:enumeration value $=" \mathrm{Ta} / />$ <xsd:enumeration value $=" \mathrm{~W} " />$ <xsd:enumeration value $=" R e " />$ <xsd:enumeration value $=" \mathrm{Os} " />$ $<x$ sd:enumeration value $="|r| />$ $<$ xsd:enumeration value $=" \mathrm{Pt} / />$ $<x$ sd:enumeration value="Au"/> $<x$ sd:enumeration value $=" \mathrm{Hg} " />$ $<x$ sd:enumeration value $=" \mathrm{TI} / \mathrm{s}$ $<x$ sd:enumeration value $=" \mathrm{~Pb} " />$ $<x$ sd:enumeration value $=" \mathrm{Bi}^{\prime \prime} />$ $<x$ sd:enumeration value="Po"/> <xsd:enumeration value $=" \mathrm{At} " />$ $<x$ sd:enumeration value $=" \mathrm{Rn} " />$ <xsd:enumeration value $=" \mathrm{Fr} " />$ $<x$ sd:enumeration value $=" \mathrm{Ra}$ "/ $/>$ $<x$ sd:enumeration value $=" \mathrm{Ac} " />$ <xsd:enumeration value="Th"/> $<x$ sd:enumeration value $=" \mathrm{~Pa} " />$ $<x$ sd:enumeration value $=" U " />$ $<x$ sd:enumeration value $=" \mathrm{~Np} " />$ $<x$ sd:enumeration value $=" \mathrm{Pu}$ "/ $>$ $<x$ sd:enumeration value $=" \mathrm{Am} " />$ $<x$ sd:enumeration value $=" \mathrm{Cm} " />$ $<x$ sd:enumeration value $=" \mathrm{Bk} " />$ <xsd:enumeration value $=" \mathrm{Cf} " />$ $<x$ sd:enumeration value="Es"/> $<x$ sd:enumeration value $=" F m " />$ <xsd:enumeration value $=" \mathrm{Md} " />$ $<x$ sd:enumeration value $=" \mathrm{No} " />$ <xsd:enumeration value $=" \mathrm{Lr} / \mathrm{s}$ <xsd:enumeration value="Rf"/> $<x$ sd:enumeration value $=" \mathrm{Db} " />$ $<x$ sd:enumeration value $=" \mathrm{Sg}^{\prime \prime} />$ <xsd:enumeration value="Bh"/> $<x$ d:enumeration value $=" \mathrm{Hs} " />$ $<x$ sd:enumeration value $=" \mathrm{Mt} " />$ <xsd:enumeration value $=$ "Uun"/> <xsd:enumeration value="Uuu"/> $<x$ sd:enumeration value="Uub"/> <xsd:enumeration value="Uuq"/> <xsd:enumeration value="Uuh"/> <xsd:enumeration value="Uuo"/> $</ x s d$ :restriction $>$

$</ x$ sd:simpleType $>$

$<$ ixsd:schemas 


\section{APPENDIX B: MatMLVersion 3.0 Schema Examples}

$<? x m l$ version="1.0" encoding="UTF-8"?>

$<!-$

MatML Version 3.0 Schema Example 1 - Structural Ceramic from an Online Materials Database

Prepared by - E.F. Begley, NIST

Source - NIST WebSCD, hto://Mww.ceramics.nist.gov/srd/scd/Z00363.htm. R.G. Munro and E.F. Begley,

January 2002

$\rightarrow$

<MatML_Doc xmlns:xsi="http://www.w3.org/2000/10/XMLSchema-instance" xsi:noNamespaceSchemaLocation="matml.xsd"> $<$ Material> $<$ BulkDetails $>$

$<$ Name $>$ silicon nitride $<$ Name $>$

$<$ Class $>$ ceramic $<$ Class $>$

$<$ Subclass $>$ nitride $</$ Subclass $>$

$<$ Specification $>$ NCX $-5102</$ Specification $>$

$<$ Source>Saint-Gobain/Norton Industrial Ceramics $</$ Source $>$

$<$ Form $>$ bar $<$ Form $>$

$<$ ProcessingDetails>

$<$ Name $>$ hot isostatic pressing $<$ Name $>$

$<$ Notes $>$

"The material produced is designated NCX-5102 and consists of a silicon nitride- $4 \%$ yttria composition that is densified by glass-encapsulation HIPing. ... Large-scale batches $(30 \mathrm{~kg})$ of Si3N $4-4 \%$ Y $2 \mathrm{O} 3$ powder were milled in water, and the slurry was used to cast hundreds of tensile rods. The starting Si3N4 powder (Ube) was derived from a dimmide process. ... The net-shape-formed bars were HIPed using glass encapsulation (ASEA Cerma AB, Robertsford, Sweden). The HIP process was optimized using pressure, time and temperatures to assure full densification and development of an elongated microstructure for desired fracture toughness..." $</$ Notes $>$

$</$ ProcessingDetails $>$

$<$ Characterization>

$<$ Formula $>$ Si3N4.4wt\%Y2O3</Formula $>$

$<$ ChemicalComposition>

$<$ Compound>

$<$ Element>

$<$ Symbol subscript="3">Si $</$ Symbol $>$

$</$ Element>

$<$ Element>

$<$ Symbol subscript="4">N</Symbol> $</$ Element>

$<$ Compound $>$

$<$ Compound $>$

$<$ Element>

$<$ Symbol subscript="2">Y $<$ Symbol>

$</$ Element>

$<$ Element>

$<$ Symbol subscript="3">0</Symbol>

$<$ Element>

$<$ Concentration $>$

$<$ Value format="integer" $>4<$ Value $>$

$<$ Units description="mass fraction"> <Unit>\%</Unit>

$<$ Units>

$<$ Concentration>

$</$ Compound $>$

$</$ ChemicalComposition $>$

$</$ Characterization $>$

<PropertyData property="pr1" technique="mt1" source="ds 1">

$<$ Data format="integer">972,561</Data>

$<$ ParameterValue parameter="pa1" format="integer" $>23,1370</$ ParameterValue $>$

$</$ PropertyData>

<PropertyData property="pr2" technique="mt1" source="ds 1">

$<$ Data format="integer">997,396</Data>

$<$ ParameterValue parameter="pa1" format="integer">23,1370</ParameterValue $>$ 
$<$ ParameterValue parameter="pa2" format="string" >540-1237,344-452</ParameterValue >

$<$ PropertyData>

$<$ PropertyData property="pr3" technique="mt1" source="ds1">

$<$ Data format="string" $>4,-,-</$ Data $>$

$<$ ParameterValue parameter="pa5" format="string" $>$ Tensile,Flexural,Flexural $</$ ParameterValue $>$

$<$ ParameterValue parameter="pa1" format="integer">23,23,1370</ParameterValue>

$<$ ParameterValue parameter="pa4" format="integer" $>665,653,517<$ ParameterValue>

$<$ ParameterValue parameter="pa6" format="integer">1109,-,-</ParameterValue>

$<$ /PropertyData>

$<$ PropertyData property="pr4" technique="mt1" source="ds1">

$<$ Data format="integer" $>1109</$ Data $>$

$<$ ParameterValue parameter="pa5" format="string" $>$ Tensile $</$ ParameterValue $>$

$<$ ParameterValue parameter="pa1" format="integer" $>23</$ ParameterValue $>$

$<$ ParameterValue parameter="pa3" format="integer" $>4</$ ParameterValue $>$

$<$ ParameterValue parameter="pa4" format="integer">665</ParameterValue>

$<$ PropertyDatas

$<$ BulkDetails $>$

$<$ Metadata>

$<$ DataSourceDetails id="ds1" type="journal article">

$<$ Name>

"Reliable Ceramics for Advanced Heat Engines," V.K. Pujari, D.M. Tracey, M.F. Foley, N.I. Paille, P.J. Pelletier,

L.C. Sales, C.A. Willkens, and R.L. Yeckley, American Ceramic Society Bulletin, Vol. 74[4], pp. 86-90

(1995), published by American Ceramic Society

$$
<\text { Name }>
$$

$<$ Notes $>$

Cautions

1. Data evaluated by acceptance criteria

2. "The nonlinear character of the distribution with multiple inflections suggests that a two-parameter Weibull

fit of these data (sigma $=1038 \mathrm{MPa}, \mathrm{m}=10.4$ ) is inappropriate and that the multimodal nature of the data should be represented using competing risk analysis. ... The important feature of the three-parameter Weibull distribution is the existence of a threshold stress below which there is zero probability of failure." $<$ Notes $>$

$</$ DataSourceDetails $>$

$<$ PropertyDetails id="pr1" type="mechanical">

$<$ Name $>$ Flexural Strength $<$ Name>

$<$ Units name="MPa" description="megapascals"> $<$ Unit $>\mathrm{MPa}<$ /Unit>

$<$ Units $>$

$<$ PropertyDetails $>$

$<$ PropertyDetails id="pr2" type="mechanical">

$<$ Name $>$ Tensile Strength $<$ Name $>$

$<$ Units name="MPa" description="megapascals"> $<$ Unit $>\mathrm{MPa}</$ Unit $>$

$<$ Units $>$

$<$ PropertyDetails>

<PropertyDetails id="pr3" type="mechanical">

$<$ Name $>$ Weibull Modulus $<$ Name $>$

$<$ Unitless/>

$<$ PropertyDetails $>$

<PropertyDetails id="pr4" type="mechanical">

$<$ Name $>$ Weibull Strength $<$ Name $>$

<Units name="MPa" description="megapascals"> $<$ Unit>MPa $<$ Unit>

$<$ Units $>$

$<$ PropertyDetails>

$<$ MeasurementTechniqueDetails id="mt1">

$<$ Name $>$ Literature survey $<$ /Name $>$

$<$ Notes>

The authors cite V.R. Pujari et al., "Development of Improved Processing and Evaluation Methods for High

Reliability Structural Ceramics for Advanced Heat Engine Applications, Phase I," final report, ORNL/Sub/89-SB182/1, NTIS Rept. No. DE93-040528, August (1993), and summarize the procedure as follows. "The cylindrical buttonhead specimens were machined to ORNL design with a gauge diameter of $6.0 \pm 0.1 \mathrm{~mm}$. ...50 mm diameter, $150 \mathrm{~mm}$ long specimens... were machined as many flexure bars $(3 \mathrm{~mm}$ by $4 \mathrm{~mm}$ by $50 \mathrm{~mm})$ for assessment of the properties across the $50-\mathrm{mm}$ section."

$<$ Notes $>$

$<$ MeasurementTechniqueDetails> 
$<$ ParameterDetails id ="pa1">

$<$ Name $>$ Test Temperature $<$ Name $>$

$<$ Units name="degree Celsius"> $<$ Unit $>{ }^{\circ} \mathrm{C}<$ /Unit $>$

$<$ Units >

$<$ ParameterDetails>

$<$ ParameterDetails id="pa2">

$<$ Name $>$ Range of Strengths $<$ Name $>$

<Units name="MPa" description="megapascals"> $<$ Unit $>\mathrm{MPa}<$ UUnit>

$<$ Units >

$<$ ParameterDetails>

$<$ ParameterDetails id="pa3">

$<$ Name $>$ Weibull Modulus $<$ Name $>$

$<$ Unitless/>

$<$ ParameterDetails>

$<$ ParameterDetails id="pa4">

$<$ Name $>$ Threshold Strength $<$ Name $>$

$<$ Units name="MPa" description="megapascals"> $<$ Unit $>\mathrm{MPa}<$ Unnit $>$

$<$ Units >

$<$ ParameterDetails>

$<$ ParameterDetails id="pa5">

$<$ Name $>$ Stress Mode $<$ Name $>$

$<$ Unitless/>

$<$ ParameterDetails>

<ParameterDetails id="pa6">

$<$ Name $>$ Weibull Strength $<$ Name $>$

$<$ Units name="MPa" description="megapascals"> $<$ Unit $>\mathrm{MPa}<$ /Unit>

$<$ Units $>$

$<$ ParameterDetails $>$

$<$ Metadata $>$

$<$ Material $>$

$<$ MatML_Doc> 
$<!--$

MatML Version 3.0 Schema Example 2 - Aluminum Alloy from a Printed Handbook

Prepared by - E.F. Begley, NIST and J.G. Kaufman, The Aluminum Association, Inc.

Source - Handbook data generously provided by F. Cverna of ASM International and J.G. Kzufman of the

Aluminum Association from Properties of Auntinum Alloys. p. 291, ASM International, Materials Park, Oho,

ISBN: 0-87170-632-6, 1999

$-\rightarrow>$

<MatML_Doc xmlns:xsi="http://www.w3.org/2000/10/XMLSchema-instance" xsi:noNamespaceSchemaLocation="matml.xsd"> $<$ Material $>$

$<$ BulkDetails>

$<$ Name authority="The Aluminum Association Alloy Designation System" $>1350<$ Name $>$

$<$ Class $>$ metal $<$ /Class $>$

$<$ Subclass >aluminum alloy</Subclass $>$

$<$ Specification $>$ ASTM B230</Specification>

$<$ Form $>$ Rolled rod and shapes $<$ /Form $>$

$<$ ProcessingDetails>

$<$ Name $>$ Temper $\mathrm{H} 18<$ Name>

$<$ ProcessingDetails $>$

$<$ Characterization>

$<$ Formula $>$ Al $</$ Formula $>$

$<$ DimensionalDetails>

$<$ Name $>$ Thickness $<$ Name $>$

$<$ Value format="string" $>0.5,2.0<$ Nalue $>$

$<$ Units name $=$ "inches" $>$

$<$ Unit>in</Unit>

$<$ Units $>$

$<$ Qualifier>min.,max.</Qualifier>

$</$ DimensionalDetails $>$

$<$ Characterization $>$

<PropertyData property="pr1" technique="mt1" source="ds1" specimen="sp1">

$<$ Data format $=$ "float" $>+23,+17,+15,+14.5,+14.5<$ /Data $>$

$<$ ParameterValue parameter="pa1" format="integer" $>0,0,0,0,0<$ ParameterValue $>$

$<$ ParameterValue parameter="pa2" format="integer">1,1,1,1,1</ParameterValue $>$

$<$ ParameterValue parameter="pa3" format="exponential" $>1.0 \mathrm{E} 5,1.0 \mathrm{E} 6,1.0 \mathrm{E} 7,1.0 \mathrm{E} 8,5.0 \mathrm{E} 8</$ ParameterValue $>$ $<$ PropertyData>

$<$ PropertyData property="pr2" technique="mt1" source="ds 1 " specimen="sp1">

$<$ Data format="integer" >+160,+115,+105,+100,+100</Data>

<ParameterValue parameter="pa1" format="integer">0,0,0,0,0</ParameterValue>

$<$ ParameterValue parameter="pa2" format="integer" $>1,1,1,1,1</$ ParameterValue $>$

$<$ ParameterValue parameter="pa3" format="exponential" >1.0E5,1.0E6,1.0E7,1.0E8,5.0E8</ParameterValue $>$

$</$ PropertyData $>$

<PropertyData property="pr1" technique="mt1" source="ds1" specimen="sp1">

$<$ Data format $=$ "float" $>+11.5,+8.5,+7,+6.5,+6.5<$ Data $>$

$<$ ParameterValue parameter="pa1" format="integer" $>-1,-1,-1,-1,-1<$ ParameterValue $>$

$<$ ParameterValue parameter="pa2" format="integer">1,1,1,1,1</ParameterValue $>$

$<$ ParameterValue parameter="pa3" format="exponential" >1.0E5,1.0E6,1.0E7,1.0E8,5.0E8</ParameterValue $>$

$<$ PropertyData>

<PropertyData property="pr2" technique="mt1" source="ds1" specimen="sp1">

$<$ Data format="integer" $>+80,+59,+48,+45,+45</$ Data $>$

$<$ ParameterValue parameter="pa1" format="integer">-1,-1,-1,-1,-1</ParameterValue $>$

$<$ ParameterValue parameter="pa2" format="integer">1,1,1,1,1</ParameterValue $>$

$<$ ParameterValue parameter="pa3" format="exponential">1.0E5,1.0E6,1.0E7,1.0E8,5.0E8 $</$ ParameterValue $>$

$<$ PropertyData>

$</$ BulkDetails $>$

$<$ Metadata>

$<$ DataSourceDetails id="ds1" type="handbook, typical data">

$<$ Name>

"Properties of aluminum alloys : tensile, creep, and fatigue data at high and low temperatures" / edited by J. Gilbert Kaufman. $<$ Names

$<$ Notes $<$ Notes $>$

Plus (+) indicates tension; minus (-) indicates compression.

$<$ DataSourceDetails> 
<PropertyDetails id="pr1" type="mechanical"> $<$ Name>Axial-Stress Fatigue Strength</Name>

$<$ Units name="ksi" description="kip per square inch"> $<$ Unit>ksi</Unit>

$<$ Units $>$

$<$ PropertyDetails $>$

$<$ PropertyDetails id="pr2" type="mechanical">

$<$ Name $>$ Axial-Stress Fatigue Strength $<$ Name $>$

$<$ Units name="MPa" description="megapascals">

$<$ Unit $>\mathrm{MPa}</$ Unit $>$

$<$ Units>

$<$ PropertyDetails>

$<$ MeasurementTechniqueDetails id="mt1">

$<$ Name>ASTM E597</Name>

$<$ MeasurementTechniqueDetails>

<SpecimenDetails id="sp1" type="cylindrical">

$<$ Name>smooth specimen</Name>

$<$ Notes $>$ diameter $=0.375$ inches $<$ Notes $>$

$</$ SpecimenDetails $>$

$<$ ParameterDetails id="pa1">

$<$ Name $>$ Stress Ratio</Name>

$<$ Unitless/>

$<$ Notes $>$ Stress Ratio $(R)=($ minimum stress $) /($ maximum stress $)<$ Notes $>$

$<$ ParameterDetails>

<ParameterDetails id="pa2">

$<$ Name $>$ Number of Samples $<$ Name $>$

$<$ Unitless/>

$<$ ParameterDetails $>$

$<$ ParameterDetails id="pa3">

$<$ Name $>$ Number of Cycles $<$ Name $>$

$<$ Unitless/>

$<$ ParameterDetails $>$

$<$ Metadata>

$<$ Glossary>

$<$ Term $>$

$<$ Name $>\mathrm{H} 18<$ Name $>$

$<$ Definition>

"H18" is a code from The Aluminum Association Temper Designation System. The H is defined as "strain-hardened (wrought products only). The 1 applies to products that are strain-hardened to obtain the desired strength without supplementary thermal treatment. The 8 indicates the degree of strain-hardening and is assigned to the hardest tempers normally produced.

$<$ Definition $>$

$<$ Term>

$<$ Term $>$

$<$ Name $>1350<$ Name $>$

$<$ Definition>

"1350" is a code from The Aluminum Association Alloy Designation System. The first digit of the code represents the principal alloying constitutent(s). The second digit indicates variations of the initial alloy. The third and fourth digits indicate individual alloy variations (the numbers have no significance but are unique). 1350 is an alloy that is pure AL $(99.00 \%$ or greater). For further details, contact The Aluminum Association, 900 19th Street, N.W., Washington, D.C. 20006.

$<$ Term>

$$
</ \text { Definition> }
$$

</Material>

<MatML_Doc> 
$<$ ?xml version $=" 1.0$ " encoding $="$ UTF-8"? $>$

$<!--$

MatML Version 3.0 Schema Example 3 - Steel with TiC Coating from a Journal Article

Prepared by - E.F. Begley, NIST

Source - A. Agamal and N.B. Dahotre, "Pulsed Electrode Surfacing of Steel With TiC Coating -

Microstructure and Wear Properties," ASM Journal of Materials Engineering and Periormance, Vol. 8 ,

No. 4, pp. 479-486, 1999

$-->$

<MatML_Doc xmins:xsi="http://www.w3.org/2000/10/XMLSchema-instance" xsi:noNamespaceSchemaLocation="matml.xsd"> $<$ Material>

$<$ BulkDetails $>$

$<$ Name $>$ TiC coated AISI 1018 steel</Name>

$<$ Class >composite</Class>

$<$ Subclass $>$ ceramic coating on metal substrate $<$ Subclass $>$

$<$ Form $>$ coupon</Form $>$

$<$ PropertyData property="pr1" technique="mt1" source="ds $1 ">$

$<$ Data format="float" $>.0011, .0018, .0023, .0027, .0029</$ Data $>$

$<$ ParameterValue parameter="pa1" format="integer" >2,4,6,8,10</ParameterValue>

$<$ ParameterValue parameter="pa2" format="integer" >270,270,270,270,270</ParameterValue>

$<$ ParameterValue parameter="pa3" format="integer">2,2,2,2,2</ParameterValue>

$<$ PropertyData>

$<$ PropertyData property="pr2" technique="mt2" source="ds1">

$<$ Data format $=$ "float" $>0.58<$ Data $>$

$<$ PropertyDatas

$<$ BulkDetails>

$<$ ComponentDetails>

$<$ Name $>$ steel $<$ Name $>$

$<$ Class $>$ metal</Class $>$

$<$ Specification authority="American Iron and Steel Institute">AISI 1018</Specification $>$

$<$ Form>coupon</Form>

$<$ ProcessingDetails>

$<$ Name $>$ Mechanical Polishing </Name>

$<$ Notes>The coupons were mechanically polished on emery paper of grit size $240 .<$ Notes>

$<$ ProcessingDetails $>$

$<$ ProcessingDetails>

$<$ Name $>$ Rinsing $<$ /Name $>$

$<$ Notes $>$ After polishing, the coupons were rinsed in acetone. $<$ Notes $>$

$<$ ProcessingDetails $>$

$<$ ProcessingDetails>

$<$ Name $>$ Coating $<$ Name $>$

$<$ Notes $>$

"A sintered electrode of $\mathrm{TiC}$ was used to deposit a coating on these steel coupons. The TiC electrode had 3 to $5 \mathrm{wt} \% \mathrm{Ni}$ and 1 to $3 \mathrm{wt} \% \mathrm{Fe}$ as binder. Deposition was carried out using a handheld gun in air at room temperature. Pulsed electrode deposition was carried out at a voltage of $50 \mathrm{~V}$ and spark time of $10 \mu \mathrm{s}$. The discharge capacitance used for the PES process was $450 \mu \mathrm{F}$ with a current of $25 \mathrm{~A}$." $<$ Notes>

$</$ ProcessingDetails $>$

$<$ Geometry>

$<$ Shape $>$ square $</$ Shape $>$

$<$ Dimensions $>25 \mathrm{~mm} \times 25 \mathrm{~mm}</$ Dimensions $>$

$<$ Geometry $>$

$<$ PropertyData property="pr1" technique="mt3" source="ds1">

$<$ Data format="float" $>.0019, .0036, .0057, .0073, .0090</$ Data $>$

$<$ ParameterValue parameter="pa1" format="integer" $>2,4,6,8,10</$ ParameterValue $>$

$<$ ParameterValue parameter="pa2" format="integer">270,270,270,270,270</ParameterValue>

$<$ ParameterValue parameter="pa3" format="integer" $>2,2,2,2,2</$ ParameterValue $>$

$</$ PropertyData>

$<$ PropertyData property="pr3" technique="mt4" source="ds1">

$<$ Data format="integer" $>172<$ Data $>$

$<$ Uncertainty>

$<$ Value format $=$ "integer" $>12<$ Value $>$

$<$ Units name="kg/mm^2" description="kilograms per square millimeter">

$<$ Unit $\mathrm{kg}</$ Unit>

$<$ Unit power="-2">mm</Unit> 
$<$ Units >

$<$ Uncertainty $>$

$<$ PropertyData>

<AssociationDetails>

<Associate>titanium carbide coating </Associate>

$<$ Relationship >substrate $</$ Relationship >

$<$ AssociationDetails>

$</$ ComponentDetails >

$<$ ComponentDetails>

$<$ Name $>$ titanium carbide coating $</$ Name $>$

$<$ Class $>$ carbide $<$ /Class $>$

$<$ Subclass $>$ monocarbide $</$ Subclass $>$

$<$ Characterization >

$<$ Formula $>\mathrm{TiC} \cdot \mathrm{xFe}</$ Formula $>$

$<$ PhaseComposition>

$<$ Name $>$ TiC $<$ /Name $>$

$<$ PhaseComposition>

$<$ PhaseComposition>

$<$ Name $>\mathrm{Ti}<$ /Name $>$

$<$ Concentration $>$

$<$ Value format="string" $>5,25<$ Value $>$

$<$ Units description="mass fraction"> $<$ Unit $\%$ </Unit>

$<$ Units>

$<$ Qualifiersmin., max.</Qualifier>

$</$ Concentration $>$

$<$ PhaseComposition>

$<$ PhaseComposition>

$<$ Name $>\mathrm{Fe}-\mathrm{C}$ (austenite) $<$ Name $>$

$<$ PhaseComposition>

$<$ PhaseComposition>

$<$ Name $>\mathrm{Fe}$ (ferrite) </Name>

$<$ PhaseComposition>

$<$ PhaseComposition>

$<\mathrm{Name}>\mathrm{FeTi}</ \mathrm{Name}>$

$<$ Notes $>$ Possible $<$ Notes $>$

$<$ PhaseComposition>

$</$ Characterization $>$

$<$ PropertyData property="pr3" technique="mt4" source $=$ "ds 1 ">

$<$ Data format="integer" $>1235<$ /Data $>$

$<$ Uncertainty>

$<$ Value format="integer" $>86<$ Value $>$

$<$ Units name="kg/mm^2" description="kilograms per square millimeter"> $<$ Unit $\mathrm{kg}<$ Unit $>$

$<$ Unit power="-2" $>$ mm $<$ Unit $>$

$<$ Units $>$

$<$ Uncertainty>

$<$ PropertyData>

$<$ AssociationDetails>

<Associate>AISI 1018 steel</Associate>

$<$ Relationship >coating </Relationship >

$<$ AssociationDetails $>$

$<$ ComponentDetails $>$

$<$ ComponentDetails>

$<$ Name $>$ heat affected zone $(\mathrm{HAZ})</$ Name $>$

$<$ PropertyData property="pr3" technique="mt4" source="ds1">

$<$ Data format="integer" $>352<$ Data $>$

$<$ Uncertainty $>$

$<$ Value format $=$ "integer" $>32<$ Value $>$

$<$ Units name $=" \mathrm{~kg} / \mathrm{mm}^{\wedge} 2^{\prime \prime}$ description="kilograms per square millimeter" $>$ $<$ Unit $>$ kg</Unit>

$<$ Unit power="-2">mm</Unit>

$<$ Units>

$<$ Uncertainty $>$

$<$ PropertyData>

$<$ Notes >Martensitic Zone</Notes $>$ 


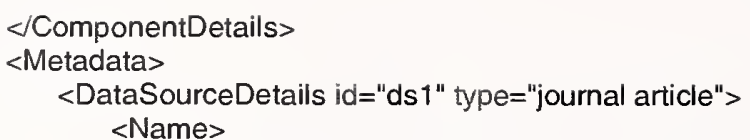

A. Agarwal and N.B. Dahotre, "Pulse Electrode Surfacing of Steel with TiC Coating: Microstructure and Wear $<$ Name $>$

Properties," ASM Journal of Materials Engineering and Performance, Vol. 8, No. 4, pp. 479-486, 1999 $<$ Notes $>$ $</$ Notes $>$

Data were digitized from Fig. 9. The reported unit, "gm", is interpreted to mean "g", grams.

$</$ DataSourceDetails $>$

$<$ PropertyDetails id="pr1" type="mechanical"> $<$ Name $>$ Wear (Weight Loss Analysis) $<$ Name>

$<$ Units name $=" g$ " description="gram"> $<$ Unit $>$ g $<$ Unit $>$ $<$ /Units $>$

$<$ PropertyDetails $>$

$<$ PropertyDetails id="pr2" type="mechanical"> $<$ Name $>$ Coefficient of Friction $<$ Name $>$ $<$ Unitless/>

$<$ PropertyDetails $>$

$<$ PropertyDetails id="pr3" type="mechanical"> $<$ Name $>$ Microhardness $<$ Name $>$

$<$ Units name=" $\mathrm{kg} / \mathrm{mm}^{\wedge} 2$ " description="kilograms per square millimeter"> $<$ Unit>kg </Unit> $<$ Unit power="-2">mm</Unit> $<$ Units $>$

$<$ PropertyDetails $>$

$<$ MeasurementTechniqueDetails id="mt1">

$<$ Name $>$ Block-on-Disk Tribometer $<$ Name $>$

$<$ Notes $>$

"Coated coupons of dimension $25 \times 25 \mathrm{~mm}$ were tested for dry sliding wear against a hardened steel ring rotating at a linear speed of $270 \mathrm{~m} / \mathrm{min}$. Weight loss measurements were made after successive $2 \mathrm{~min}$. The dry sliding wear test was conducted for 10 min with an applied normal load of 2 kg." $<$ Notes $>$

$<$ MeasurementTechniqueDetails $>$

$<$ MeasurementTechniqueDetails id="mt2">

$<$ Name $>$ Block-on-Disk Tribometer $<$ Name $>$

$<$ Notes $>$

"The coefficient of friction $(\mu)$ was also recorded simultaneously by an interface computer, which acquired data in the form of electrical output power of the motor. Even though data were recorded at a frequency of $1 \mathrm{~Hz}$ for a total test time of $10 \mathrm{~min}$, an average of 10 successive points was taken for computing the coefficient of friction, $\mu$....the coefficient of friction is calculated by measuring the changes in voltage and current in the electrical circuit of the motor driving the block-on-ring tribometer during loading..." $<$ Notes $>$

$<$ MeasurementTechniqueDetails $>$

$<$ MeasurementTechniqueDetails id="mt3"> $<$ Name $>$ Block-on-Disk Tribometer $<$ Name>

$<$ MeasurementTechniqueDetails>

$<$ MeasurementTechniqueDetails id="mt4">

$<$ Name $>$ Knoop Indentation $</$ Name $>$

$<$ Notes $>$

"Microhardness measurements were performed on a Buehler Micromet II microhardness tester using a Knoop indenter with normal load of $200 \mathrm{~g}$ applied for $15 \mathrm{~s}$." $<$ Notes $>$

$<$ MeasurementTechniqueDetails>

$<$ ParameterDetails id="pa1">

$<$ Name $>$ Time $<$ /Name $>$

$<$ Units name $=$ "min" description="minutes"> $<$ Unit $>\min </$ Unit $>$

$</$ Units $>$

$<$ ParameterDetails>

$<$ ParameterDetails id="pa2">

$<$ Name>Sliding Speed (Steel Ring)</Name>

$<$ Units name="m/min" description="meters per minute"> $<$ Unit $>$ m $<$ /Unit $>$ 

$<$ Units>

$<$ Unit power=" -1 " $>\min <$ Unit $>$

$<$ ParameterDetails>

<ParameterDetails id="pa3">

<Name>Applied Normal Load</Name>

$<$ Units name="kg" description="kilograms"> $<$ Unit $>\mathrm{kg}<$ /Unit> $<$ Units>

$<$ ParameterDetails $>$

$<$ Metadata $>$

$<$ Material>

$<$ MatML_Doc> 


